\title{
A Method on Estimating Time-Varying Vertical Eddy Viscosity for an Ekman Layer Model with Data Assimilation
}

\author{
JICAI ZHANG \\ Institute of Physical Oceanography, Ocean College, Zhejiang University, Zhoushan, China, and Applied Ocean \\ Physics and Engineering Department, Woods Hole Oceanographic Institution, Woods Hole, Massachusetts \\ GUOQING LI AND JIACHENG YI \\ Institute of Physical Oceanography, Ocean College, Zhejiang University, Zhoushan, China \\ YANQIU GAO \\ State Key Laboratory of Satellite Ocean Environment Dynamics, Second Institute of Oceanography, \\ Ministry of Natural Resources, Hangzhou, China \\ ANZHOU CAO \\ Institute of Physical Oceanography, Ocean College, Zhejiang University, Zhoushan, China
}

(Manuscript received 11 December 2018, in final form 7 July 2019)

\begin{abstract}
Temporal vertical eddy viscosity coefficient (VEVC) in an Ekman layer model is estimated using an adjoint method. Twin experiments are carried out to investigate the influences of several factors on inversion results, and the conclusions of twin experiments are 1) the adjoint method is a capable method to estimate different kinds of temporal distributions of VEVCs; 2) the gradient descent algorithm is better than CONMIN and L-BFGS for the present problem, although the posterior two algorithms perform better on convergence efficiency; 3 ) inversion results are sensitive to initial guesses; 4) the model is applicable to different wind conditions; 5) the inversion result with thick boundary layer depth (BLD) is slightly better than thin BLD; 6) inversion results are more sensitive to observations in upper layers than those in lower layers; 7) inversion results are still acceptable when data noise exists, indicating the method can sustain noise to a certain degree; 8) a regularization method is proved to be useful to improve the results for present problem; and 9) the present method can tolerate the existence of balance errors due to the imperfection of governing equations. The methodology is further validated in practical experiments where Ekman currents are derived from Bermuda Testbed Mooring data and assimilated. Modeled Ekman currents coincide well with observed ones, especially for upper layers. The results demonstrate that the assumptions of depth dependence and time dependence are equally important for VEVCs. The feasibility of the typical Ekman model, the imperfection of Ekman balance equations, and the deficiencies of the present method are discussed. This method provides a potential way to realize the time variations of VEVCs in ocean models.
\end{abstract}

\section{Introduction}

The effect of oceanic vertical eddy viscosity (VEV), which is closely related to the turbulence, plays an important role in ocean motions. Lentz (1995) demonstrated the sensitivity of the cross-shelf circulation over the inner shelf, especially the location and width of the cross-shelf divergence in the Ekman transport, to the form of the

Corresponding author: Jicai Zhang, jicai_zhang@163.com
VEV profile. McWilliams et al. (2009) and Wirth (2010) investigated the influence of vertical structure of the eddy viscosity on the Ekman spiral structure. How to parameterize the effect of turbulence is of great importance for oceanic studies (Davies and Xing 2001). Compared with large-eddy simulation (LES) and direct numerical simulation (DNS), the Reynolds-averaged Navier-Stokes equations (RANS) can be implemented with minor computation cost, which makes it the most popular method to depict the turbulence, especially for the motions 
with large Reynolds numbers, including oceanic and atmospheric processes. Turbulence models are needed to close the equations and calculate the Reynolds stress and turbulent scalar flux. Many turbulence models have been developed, for example, the Prandtl's mixing length model, $k-\varepsilon$ model, Mellor-Yamada model, Reynolds stress model, and KPP model. (Obermeier 2006; Mellor and Yamada 1982; Launder et al. 1975; Large et al. 1994). Unfortunately, there is no such model that can fit turbulent flows under different kinds of situations. One of the largest deficiencies of the RANS method is the lack of universality. How to increase the universality of RANS method is essential to depicting the effects of turbulence.

The vertical eddy viscosity coefficients (VEVCs), which cannot be measured directly, are an important parameter in ocean models and usually assigned by experience or calculated by turbulence models. In many earlier works, the coefficient was regarded as a constant in space and time domain, which is easy to perform but not accurate. The VEVCs produced by turbulence models are more reasonable by taking into account ocean dynamics. However, there are many parameters that need to be assigned for most of the complex turbulence models. It is usually difficult to decide these parameters, especially when turbulence observations are absent or inadequate, which has significantly limited the application and accuracy of turbulence models. Besides, turbulent flows are quite complex with time and space scales spanning a wide range, and the nonuniversality of turbulence models, as indicated above, also becomes a block.

Data assimilation methods, especially the complex ones like four-dimensional variational data assimilation (4DVAR), are developed on the basis of rigorous mathematical theories, such as inverse problem theory and optimal control theory (Thacker and Long 1988). The ultimate purpose of applying data assimilation method is to reduce the data misfit between model results and various observations, by either estimating the model parameters or dynamically interpolating the observations (Anderson et al. 1996). Among all the data assimilation methods, 4DVAR is one of the most effective and powerful approaches (Kazantsev 2012). As one of 4DVAR, the adjoint method is an advanced data assimilation technique and has the advantage of directly assimilating various observations distributed in time and space into numerical models, while maintaining dynamical and physical consistency with the model (Zhang and Lu 2010). The adjoint method is a powerful tool for parameter estimation (Navon et al. 1992; Navon 1998). Navon (1998) presented a significant overview of parameter estimation in meteorology and oceanography in view of applications of 4DVAR data assimilation techniques to inverse parameter estimation problems.

As a type of inverse problem, the methodology of parameter estimation has been applied to determine VEVCs (Panchang and Richardson 1993; Lardner and Song 1995). Using adjoint method, Yu and O'Brien (1991), Zhang et al. (2009), and Cao et al. (2017) estimated the depthdependent VEVCs by assimilating in situ or pseudo measurements. Using the least squares method, Yoshikawa and Endoh (2015) estimated the tide-induced eddy viscosity profile by solving the Ekman balance equations with measured velocity spirals. However, previous research mostly considered the VEVCs as a constant or depth dependent. The ocean is a time-varying system and the parameters decided by ocean dynamics should also be time varying. For example, the surface drag coefficients will change with time under different wind conditions (Jarosz et al. 2007; Maurer et al. 2015). The bottom friction coefficients and other parameters also have the similar variability in the time domain (Lacy et al. 2005; Lozovatsky et al. 2008; Gwyther et al. 2015). The VEVCs can be influenced by heat transferring processes as well as vertical mixing induced by surface and bottom friction (Pohlmann 1996), upwelling/downwelling (Kirincich and Barth 2009), cycle of spring and neap tides (Yoshikawa et al. 2010), etc. If time-varying VEVCs are applied in ocean numerical models, the accuracy of simulation can be improved, which activates the present work. As one of the most important theories in oceanography, whether the typical Ekman theory can be observed in the real ocean has been studied continuously (Price et al. 1987; Chereskin 1995; Elipot and Gille 2009; Lenn and Chereskin 2009; Polton et al. 2013; Roach et al. 2015). These studies found the classical constant viscosity inadequate and in need of improvements. To improve the model, this work aims to develop a methodology of estimating the time-varying VEVCs from in situ current observations by applying an adjoint data assimilation method.

The organization of this paper is as follows. The numerical model is shown in section 2 . In section 3, identical twin experiments are performed to determine the optimum settings for the method. In section 4 , the method is further validated in practical experiments where in situ Ekman currents are assimilated. The feasibility, the imperfection of Ekman balance equations, and deficiencies of the present method is discussed in section 5. Conclusions are given in section 6 .

\section{Numerical model}

\section{a. Governing equations (forward model)}

Ekman layer model is a starting point for studying upper-ocean physics and has been applied in numerous 
works (Elipot and Gille 2009). In this model, a horizontally unbounded ocean surface layer with depth $H_{0}$ is considered. The $z$ axis points upward, and the sea surface locates at $z=0$. The Coriolis parameter $f$ is a constant ( $f$-plane assumption). Governing equations are shown as follows:

$$
\left\{\begin{array}{l}
\frac{\partial u}{\partial t}-f v=\frac{\partial}{\partial z}\left(A \frac{\partial u}{\partial z}\right) \\
\frac{\partial v}{\partial t}+f u=\frac{\partial}{\partial z}\left(A \frac{\partial v}{\partial z}\right) .
\end{array}\right.
$$

The corresponding boundary conditions are

$$
\begin{gathered}
\left.\frac{\partial u}{\partial z}\right|_{z=0}=\frac{C_{D}}{A} \frac{\rho_{a}}{\rho_{w}} \sqrt{u_{a}^{2}+v_{a}^{2}} u_{a}, \\
\left.\frac{\partial v}{\partial z}\right|_{z=0}=\frac{C_{D}}{A} \frac{\rho_{a}}{\rho_{w}} \sqrt{u_{a}^{2}+v_{a}^{2}} v_{a}, \\
\left.A \frac{\partial u}{\partial z}\right|_{z=-H_{0}}=0,\left.\quad A \frac{\partial v}{\partial z}\right|_{z=-H_{0}}=0 .
\end{gathered}
$$

The initial conditions are

$$
\left.u\right|_{t=0}=u_{0},\left.\quad v\right|_{t=0}=u_{0},
$$

where $u(z)$ (positive to the east) and $v(z)$ (positive to the north) are horizontal velocity components; $u_{a}$ and $v_{a}$ are eastward and northward wind velocity components, respectively; $\rho_{a}$ and $\rho_{w}$ are densities of air and water, respectively; $C_{D}$ is the wind stress drag coefficient; and $A(t)$ is the VEVCs. Assuming that the wind force is only along the $u$ direction, $v_{a}$ is 0 .

Considering that the variables in governing equations have different units and magnitudes, the nondimensional variables are introduced to make all the variables in the scaled problem of order unity (Yu and O'Brien 1991). The inertial period is chosen as the time scale as the motions in the upper ocean are dominated by the inertial oscillations. According to Yu and O'Brien (1991), the following nondimensional variables are introduced as

$$
\begin{gathered}
t^{\prime}=\frac{t}{T_{f}}, \quad\left(u^{\prime}, v^{\prime}\right)=\frac{(u, v)}{U}, \quad z^{\prime}=\frac{z}{D}, \\
A^{\prime}=\frac{A}{S_{a}}, \quad C_{D}^{\prime}=\frac{C_{D}}{S_{c}}, \quad\left(u_{a}^{\prime}, v_{a}^{\prime}\right)=\frac{\left(u_{a}, v_{a}\right)}{U_{a}}
\end{gathered}
$$

where

$$
T_{f}=\frac{1}{f}, \quad D=\left(\frac{s_{a}}{f}\right)^{1 / 2}, \quad U=\left(\frac{\rho_{a}}{\rho_{w}} s_{c}\right) \frac{U_{a}^{2}}{\sqrt{s_{a} f}},
$$

where $\rho_{a}=1.2 \mathrm{~kg} \mathrm{~m}^{-3}, \rho_{w}=1.025 \times 10^{3} \mathrm{~kg} \mathrm{~m}^{-3}, S_{a}=$ $5.0 \times 10^{-2} \mathrm{~m}^{2} \mathrm{~s}^{-1}, S_{c}=1.2 \times 10^{-3}$.
Equations (1)-(4) are then modified as follows:

$$
\left\{\begin{array}{l}
\frac{\partial u}{\partial t}-v=\frac{\partial}{\partial z}\left(A \frac{\partial u}{\partial z}\right) \\
\frac{\partial v}{\partial t}+u=\frac{\partial}{\partial z}\left(A \frac{\partial v}{\partial z}\right)
\end{array}\right.
$$

The corresponding boundary conditions are

$$
\begin{gathered}
\left.\frac{\partial u}{\partial z}\right|_{z=0}=C_{D} \sqrt{u_{a}^{2}+v_{a}^{2}} u_{a}, \\
\left.\frac{\partial v}{\partial z}\right|_{z=0}=C_{D} \sqrt{u_{a}^{2}+v_{a}^{2}} v_{a}, \\
\left.A \frac{\partial u}{\partial z}\right|_{z=-H_{0}}=0,\left.\quad A \frac{\partial v}{\partial z}\right|_{z=-H_{0}}=0 .
\end{gathered}
$$

The initial conditions are

$$
\left.u\right|_{t=0}=u_{0},\left.\quad v\right|_{t=0}=u_{0},
$$

In the work of Zhang et al. (2009) and Zhang et al. (2015), the VEVCs are supposed to be depth dependent and constant in time domain. It will be much more reasonable to suppose the VEVCs to be vertically and temporally varying synchronously. However, because parameter estimation belongs to the field of inverse problem, this assumption will significantly enhance the illposedness, which can lead to estimation errors. In addition, observations sampled in the Southern Ocean proved that a depth-constant VEVC can adequately represent the vertical structure of the Ekman currents, indicating a depth-constant VEVCs can be applicable in certain areas (Polton et al. 2013; Roach et al. 2015). Therefore, in this study the VEVCs are supposed to be time-varying but depth-constant values. The effect of stratification and imperfection of balance equations on the present method are discussed in section 5 .

\section{b. Adjoint model (inverse model)}

A cost function which quantifies the discrepancy between modeling results and observations is defined as

$$
J(u, v, A)=\frac{1}{2} \mathbf{K}_{m} \int_{t} \int_{z}\left[(u-\hat{u})^{2}+(v-\hat{v})^{2}\right] d z d t,
$$

where $\hat{u}$ and $\hat{v}$ denote the observed velocities, and $\mathbf{K}_{m}$ is the weighting matrix. Generally, $\mathbf{K}_{m}$ should be the inverse of the covariance matrix of observation errors, which can be simplified by assuming that observation errors are uncorrelated and equally weighted. In this study, $\mathbf{K}_{m}$ is set as a unit matrix (Zhang and Lu 2010). 
By introducing Lagrange multipliers, which are also called adjoint variables, the Lagrange function is given by

$$
\begin{aligned}
L(u, v, A, \lambda, \mu)= & J+\int_{t} \int_{z}\left\{\lambda\left[\frac{\partial u}{\partial t}-v-\frac{\partial}{\partial z}\left(A \frac{\partial u}{\partial z}\right)\right]\right. \\
& \left.+\mu\left[\frac{\partial v}{\partial t}+u-\left(A \frac{\partial v}{\partial z}\right)\right]\right\} d z d t
\end{aligned}
$$

where $\lambda$ and $\mu$ are Lagrange multipliers for $u$ and $v$, respectively. The problem of minimizing the cost function under the constraint of governing equations becomes a problem of finding stationary points of $u, v, A, \lambda$, and $\mu$ under the condition that gradients of Lagrange function vanish, which yields the following equations:

$$
\begin{array}{ll}
\frac{\partial L(u, v, A, \lambda, \mu)}{\partial \lambda}=0, & \frac{\partial L(u, v, A, \lambda, \mu)}{\partial \mu}=0 ; \\
\frac{\partial L(u, v, A, \lambda, \mu)}{\partial u}=0, & \frac{\partial L(u, v, A, \lambda, \mu)}{\partial v}=0 ; \\
\frac{\partial L(u, v, A, \lambda, \mu)}{\partial A}=0 . &
\end{array}
$$

Equations (11) recover the original governing equations, while the equations in (12) result in the adjoint equations, given by

$$
\left\{\begin{array}{l}
\frac{\partial \lambda}{\partial t}-\mu+\frac{\partial}{\partial z}\left(A \frac{\partial \lambda}{\partial z}\right)=\mathbf{K}_{m}(u-\hat{u}), \\
\frac{\partial \mu}{\partial t}+\lambda+\frac{\partial}{\partial z}\left(A \frac{\partial \mu}{\partial z}\right)=\mathbf{K}_{m}(v-\hat{v}) .
\end{array}\right.
$$

The corresponding boundary conditions are

$$
\begin{gathered}
\left.\frac{\partial \lambda}{\partial z}\right|_{z=0}=0,\left.\quad \frac{\partial \mu}{\partial z}\right|_{z=0}=0 \\
\left.\frac{\partial \lambda}{\partial z}\right|_{z=-H_{0}}=0,\left.\quad \frac{\partial \mu}{\partial z}\right|_{z=-H_{0}}=0 .
\end{gathered}
$$

The corresponding initial conditions are

$$
\left.\lambda\right|_{t=T}=0,\left.\quad \mu\right|_{t=T}=0,
$$

where $T$ is the total integral time of Ekman layer model. Ekman currents are simulated by integrating the forward model, while the adjoint variables are obtained by backward integrating the adjoint model. In general, the backward integral time of the adjoint model is equal to the integral time of the forward model (Zhang and Lu 2010). The control variable in the model (VEVC) can be optimized with (13) by applying optimization algorithms.

\section{c. Discretization of model equations}

Following Crank-Nicholson scheme, the numerical model is formulated using a finite difference discretization with a spatial increment $\Delta z$ and a temporal increment $\Delta t$. Finite difference schemes corresponding to governing equations are

$$
\begin{aligned}
& \frac{u_{j}^{i+1}-u_{j}^{i}}{\Delta t}-\frac{v_{j}^{i+1}+v_{j}^{i}}{2} \\
& =\frac{A^{i}}{2}\left(\frac{u_{j+1}^{i+1}-2 u_{j}^{i+1}+u_{j-1}^{i+1}}{\Delta z^{2}}+\frac{u_{j+1}^{i}-2 u_{j}^{i}+u_{j-1}^{i}}{\Delta z^{2}}\right), \\
& \frac{v_{j}^{i+1}-v_{j}^{i}}{\Delta t}+\frac{u_{j}^{i+1}+u_{j}^{i}}{2} \\
& =\frac{A^{i}}{2}\left(\frac{v_{j+1}^{i+1}-2 v_{j}^{i+1}+v_{j-1}^{i+1}}{\Delta z^{2}}+\frac{v_{j+1}^{i}-2 v_{j}^{i}+v_{j-1}^{i}}{\Delta z^{2}}\right) .
\end{aligned}
$$

With boundary conditions (6) and (7), the recursive relation was obtained to solve the initial boundary value problem (5)-(8):

$$
\mathbf{M} \mathbf{W}_{i}+\mathbf{M}_{0} \mathbf{W}_{i-1}+\mathbf{F}_{i}=0
$$

where

$$
\begin{aligned}
\mathbf{W} & =\left(u_{1}, u_{2}, \ldots, u_{20} ; v_{1}, v_{2}, \ldots, v_{20}\right)^{\mathrm{T}}, \\
\mathbf{F}_{i} & =\left[f_{1}(i), 0, \ldots, 0 ; f_{21}(i), 0, \ldots, 0\right]^{\mathrm{T}}, \\
f_{1}(i) & =\frac{C_{D}}{A} \Delta z \sqrt{u_{a}^{2}(i \Delta t)+v_{a}^{2}(i \Delta t)} u_{a}(i \Delta t), \\
f_{21}(i) & =\frac{C_{D}}{A} \Delta z \sqrt{u_{a}^{2}(i \Delta t)+v_{a}^{2}(i \Delta t)} v_{a}(i \Delta t) .
\end{aligned}
$$

The nonzero elements of $\mathbf{M}$ are

$$
\begin{aligned}
a_{1,1} & =1, \quad a_{1,2}=-1, \\
a_{j, j-1} & =0.45 A, \quad a_{j, j}=-1-0.9 A, \\
a_{j, j+1} & =0.45 A, \quad a_{j, j+20}=0.5 \Delta t, \quad j=2, \ldots, 19, \\
a_{20,19} & =-1, \quad a_{20,20}=1 ; \\
a_{21,21} & =1, \quad a_{21,22}=-1, \\
a_{j, j-1} & =0.45 A, \quad a_{j, j}=-1-0.9 A, \\
a_{j, j+1} & =0.45 A, \quad a_{j, j-20}=-0.5 \Delta t, \quad j=22, \ldots, 39, \\
a_{40,39} & =-1, \quad a_{40,40}=1 .
\end{aligned}
$$

The nonzero elements of $\mathbf{M}_{0}$ are 


$$
\begin{aligned}
a_{j, j-1} & =0.45 A, \quad a_{j, j}=1-0.9 A \\
a_{j, j+1} & =0.45 A, \quad j=2, \ldots, 19,22, \ldots, 39 ; \\
a_{j, j+20} & =0.5 \Delta t, \quad j=2, \ldots, 19 ; \\
a_{j, j-20} & =-0.5 \Delta t, \quad j=22, \ldots, 39 .
\end{aligned}
$$

Similar to (20), the recursive relation to solve the initial boundary value problem (14)-(17) is

$$
\mathbf{M}^{a} \mathbf{W}_{\lambda i}+\mathbf{M}_{0}^{a} \mathbf{W}_{\lambda i+1}-\mathbf{F}_{i}^{a}=0 .
$$

where

$$
\begin{aligned}
\mathbf{W}_{\lambda} & =\left(\lambda_{1}, \lambda_{2}, \ldots, \lambda_{20} ; \mu_{1}, \mu_{2}, \ldots, \mu_{20}\right)^{\mathrm{T}}, \\
\mathbf{F}_{i}^{a} & =\left[f_{1}^{a}(i), f_{2}^{a}(i), \ldots, f_{20}^{a}(i) ; f_{21}^{a}(i), f_{22}^{a}(i), \ldots, f_{40}^{a}(i)\right]^{\mathrm{T}}, \\
f_{1}^{a}(i) & =f_{20}^{a}(i)=0, \quad f_{j}^{a}(i)=\Delta t \mathbf{K}_{m}[u(i, j)-\hat{u}(i, j)], \quad j=2, \ldots, 19, \\
f_{21}^{a}(i) & =f_{40}^{a}(i)=0, \quad f_{j}^{a}(i)=\Delta t \mathbf{K}_{m}[v(i, j)-\hat{v}(i, j)], \quad j=22, \ldots, 39 .
\end{aligned}
$$

The nonzero elements of $\mathbf{M}^{a}$ are

$$
\begin{aligned}
a_{1,1} & =1, \quad a_{1,2}=-1, \\
a_{j, j-1} & =0.45 A, \quad a_{j, j}=-1-0.9 A, \\
a_{j, j+1} & =0.45 A, \quad a_{j, j+20}=-0.5 \Delta t, \quad j=2, \ldots, 19, \\
a_{20,19} & =-1, \quad a_{20,20}=1 ; \\
a_{21,21} & =1, \quad a_{21,22}=-1, \\
a_{j, j-1} & =0.45 A, \quad a_{j, j}=-1-0.9 A, \\
a_{j, j+1} & =0.45 A, \quad a_{j, j-20}=0.5 \Delta t, \quad j=22, \ldots, 39, \\
a_{40,39} & =-1, \quad a_{40,40}=1 .
\end{aligned}
$$

The nonzero elements of $\mathbf{M}_{0}^{a}$ are

$$
\begin{aligned}
a_{j, j-1} & =0.45 A, \quad a_{j, j}=1-0.9 A, \\
a_{j, j+1} & =0.45 A, \quad j=2, \ldots, 19,22, \ldots, 39 ; \\
a_{j, j+20} & =-0.5 \Delta t, \quad j=2, \ldots, 19 ; \\
a_{j, j-20} & =+0.5 \Delta t, \quad j=22, \ldots, 39 .
\end{aligned}
$$

In the matrix, index $j=1-20$ is for $u$ component, and index $j=21-40$ is for $v$ component. In (22) and (26), the values of $a_{1, j}, a_{20, j}, a_{21, j}$, and $a_{40, j}(j=40)$ have to be assigned according to the surface and boundary conditions as shown in (6) and (7), respectively.

\section{d. Optimization of the VEVCs}

Using (13), the gradient of Lagrange function $L$ with respect to $A$ yields

$$
\frac{\partial J}{\partial A}+\int_{t} \int_{z}\left(\frac{\partial \lambda}{\partial z} \frac{\partial u}{\partial z}+\frac{\partial \mu}{\partial z} \frac{\partial v}{\partial z}\right) d z d t=0
$$

Using optimization algorithm, the VEVCs can be optimized as

$$
A_{n+1}=A_{n}+\alpha_{n} d_{n},
$$

where $A_{n}$ and $A_{n+1}$ are a priori and adjusted values of the VEVCs in the $n$th iteration step, and $\alpha_{n}$ and $d_{n}$ represent the iteration step length and the search direction, respectively. In this paper, $\alpha_{n}$ is taken as $4.0 \times 10^{-4}$, which is obtained through a trial and error procedure (Hülsmann et al. 2010). There are many feasible optimization algorithms to determine $d_{n}$ (Navon and Legler 1987; Zhu and Navon 1999). Three algorithms, the gradient descent method (GD), the limited-memory conjugate gradient (CG) algorithm (CONMIN; Shanno and Phua 1980), and the limited-memory BroydenFletcher-Goldfarb-Shanno (L-BFGS; Liu and Nocedal 1989) are employed and compared in section $3 b$.

In GD, the search direction $d_{n}^{+}$is

$$
d_{n}^{+}=-g_{n}
$$

where

$$
\begin{aligned}
g_{n} & =\left(\frac{\partial J}{\partial A}\right)_{n}^{0}=\frac{(\partial J / \partial A)_{n}}{\left\|(\partial J / \partial A)_{n}\right\|}, \\
\left\|\left(\frac{\partial J}{\partial A}\right)_{n}\right\| & =\left\{\left[\left(\frac{\partial J}{\partial A}\right)_{n}\right]^{\mathrm{T}}\left(\frac{\partial J}{\partial A}\right)_{n}\right\}^{1 / 2} \\
& =\left\{\sum_{i=1}^{n}\left[\left(\frac{\partial J}{\partial A}\right)_{n}\right]^{2}\right\}^{1 / 2},
\end{aligned}
$$

where $(\partial J / \partial A)_{n}$ and $\left\|(\partial J / \partial A)_{n}\right\|$ are the gradients of the cost function with respect to the VEVCs and their $L_{2}$ norm at the $n$th iteration, respectively.

CONMIN algorithm is a limited-memory conjugate gradient method. Navon and Legler (1987) gave details on different conjugate gradient algorithms. In the conjugate gradient method, the search direction $d_{n}^{*}$ is defined as a conjugate direction: 


$$
d_{n}^{*}=-g_{n}+\frac{\left\|g_{n}\right\|^{2}}{\left\|g_{n-1}\right\|^{2}} d_{n-1} .
$$

L-BFGS is a modified BFGS algorithm with a limited amount of computer memory (Liu and Nocedal 1989;
Zou et al. 1993). It requires that the search direction $d_{n}^{\dagger}$ satisfies

$$
d_{n}^{\dagger}=-H_{n} g_{n},
$$

where

$$
\begin{aligned}
H_{n}= & \left(V_{n-1}^{\mathrm{T}}, \ldots, V_{n-m}^{\mathrm{T}}\right) H_{0}\left(V_{n-m}, \ldots, V_{n-1}\right) \\
& +\rho_{n-m}\left(V_{n-1}^{\mathrm{T}}, \ldots, V_{n-m+1}^{\mathrm{T}}\right) s_{n-m} s_{n-m}^{\mathrm{T}}\left(V_{n-m+1}, \ldots, V_{n-1}\right) \\
& +\rho_{n-m+1}\left(V_{n-1}^{\mathrm{T}}, \ldots, V_{n-m+2}^{\mathrm{T}}\right) s_{n-m+1} s_{n-m+1}^{\mathrm{T}}\left(V_{n-m+2}, \ldots, V_{n-1}\right) \\
& +\cdots \\
& +\rho_{n-1} s_{n-1} s_{n-1}^{\mathrm{T}} .
\end{aligned}
$$

where $\rho_{n}=1 / s_{n} y_{n}^{\mathrm{T}}, V_{n}=1-\rho_{n} y_{n} s_{n}^{\mathrm{T}}, s_{n}=A_{n+1}-A_{n}=$ $\alpha_{n} d_{n}, y_{n}=g_{n+1}-g_{n}$, and $m$ is the correction steps varied between three and seven (Alekseev et al. 2009). The $m$ is taken as five in this study.

For inverse problems, it is a challenge to find the global minimum of cost function. The Hessian matrix of cost function with respect to the control variables can be used to analyze whether the global minima has been reached. The Hessian matrix can be obtained by carrying out second-order adjoint (SOA) sensitivity analysis (Wang et al. 1992; Wang et al. 1995; Alekseev and Navon 2001). Wang et al. (1995) proposed a modified version of the truncated-Newton algorithm by using SOA technique to obtain an exact Hessian vector product which was required in calculating the Newton line search direction. Their method was tested in shallow water equations and obtained improved convergence efficiency compared with the original truncated-Newton method and L-BFGS. In this work, the SOA model is not developed. Nevertheless, in order to decrease the cost function continuously, the gradient of cost function with respect to control variables is normalized first, which means that only the direction of the gradient is used when optimization is performed. Then a small value is assigned to the step size $\alpha_{n}$. With this method, the cost function can be decreased without fluctuation to reach the global minimum continuously.

\section{Ideal twin experiments and results analysis}

\section{a. Model settings}

To evaluate the reasonability and feasibility of the model and method developed in this paper, ideal twin experiments are designed to estimate the time-varying VEVCs. Nine groups of experiments are carried out to investigate the factors that can influence the parameter estimation, including the optimization algorithm, initial guess, prescribed distribution, wind condition, boundary layer depth (BLD), vertical level of observations, data noise, regularization algorithm, and balance error.

The process of ideal twin experiments is designed as follows:

1) Run the forward model with prescribed VEVCs and the simulated currents are taken as pseudo "observations."

2) Run the forward model with a constant initial guess of the VEVC. Because the initial guess is different from the prescribed VEVCs, differences exist between simulated currents and observations, which act as the force of the adjoint model.

3) Based on simulated results of forward and adjoint models, the gradients of cost function with respect to VEVCs can be calculated according to (28). By employing an optimization algorithm, the VEVCs can be optimized.

4) By iterating processes 2 and 3, the VEVCs can be optimized continuously and the difference between simulated currents and observations will be reduced. Once a certain convergence criterion is met, the processes of estimation will be terminated.

In this study, unless specified, simulated velocities at all the layers are used, which means the vertical resolution of observations is equal to the spatial increment of the model $(\Delta z=5 \mathrm{~m})$. In practice, the data may be not available at some layers, or the resolution of in situ measurements is smaller than the model. However, it is not necessary for this method to use observations at every layer (Yu and O'Brien 1991). Other vertical resolutions have also been tested, such as 10.00 and $20.00 \mathrm{~m}$, but the difference can be neglected.

If there is no special specification, model parameters are set as follows:

- $f=10^{-4} \mathrm{~s}^{-1}$ for midlatitude $\left(43^{\circ} \mathrm{N}\right)$;

- $u_{0}(j)=0.01 \cos [\pi / 4-(j-0.5) \pi / 20] e^{-(j-0.5) \pi / 20} \mathrm{~m} \cdot \mathrm{s}^{-1}$ $v_{0}(j)=0.01 \sin [\pi / 4-(j-0.5)-\pi / 20] e^{-(j-0.5) \pi / 20} \mathrm{~m} \cdot \mathrm{s}^{-1}$, for generating centimeter-scale initial velocity; 

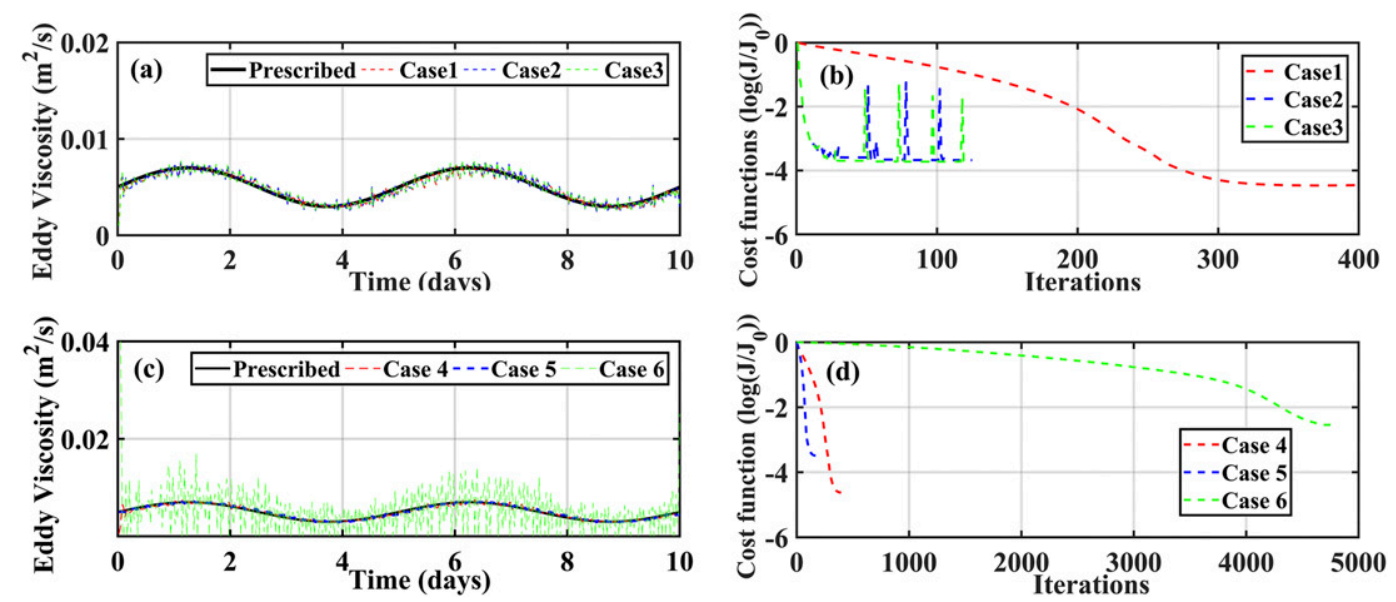

FIG. 1. (a) Prescribed and inverted VEVCs in Group 1, (b) cost functions in Group 1, (c) prescribed and inverted VEVCs in Group 2, and (d) cost functions in Group 2.

- $u_{a}=10 \sin \left(2 \pi i \Delta t / T_{0}\right) \mathrm{m} \cdot \mathrm{s}^{-1}$, where $T_{0}$ is the cycle period of winds, for generating wind speeds that vary periodically;

- $A(i)=0.005 \times\left[1+0.4 \sin \left(2 \pi i \Delta t / 12 T_{0}\right)\right] \mathrm{m}^{2} \mathrm{~s}^{-1}$ for VEVCs that vary periodically, which is regarded as the prescribed distribution of true values;

- $A_{1}=0.001 \mathrm{~m}^{2} \mathrm{~s}^{-1}$ is used as the initial guess;

- $C_{D}=1.2 \times 10^{-3}, H_{0}=100.00 \mathrm{~m}, \Delta z=5.00 \mathrm{~m}, \Delta t=$ $0.5 \mathrm{~h}, T_{0}=10 \mathrm{~h}$, and $T=10$ days.

\section{b. Group 1: Optimization algorithm}

Previous works have developed many optimization algorithms. For example, there are many forms of CG (Hager and Zhang 2005). Among all the algorithms, CONMIN (CG), GD, and L-BFGS are the most commonly used. Many studies have compared the performance of these algorithms in parameter estimation. Zou et al. (1993) carried out a study with several limitedmemory quasi-Newton methods, and concluded that L-BFGS had the best performance for some large-scale problems in oceanography and meteorology. Nevertheless, Zhang and Wang (2014) concluded that the efficiency of the L-BFGS was better than that of the GD for simple conditions in two-dimensional tidal models. Jin et al. (2015) stated that inversion results using the L-BFGS were closer to prescribed values than those using GD. However, Lu and Zhang (2006) and Zhang et al. (2011) found that GD was more efficient in the estimation of spatially varying bottom friction coefficient in a tidal model. Chen et al. (2013) demonstrated that the performance of the L-BFGS was not satisfactory when applied in practical experiments for an internal tidal model, while GD was much steadier. Zhang et al. (2015) found that the errors using GD were smaller than those using CONMIN and L-BFGS for an Ekman model. Overall, from previous works it can be concluded that none of these algorithms could be always the most effective for different problems. Liu and Nocedal (1989) found that L-BFGS with dynamic scaling performed better than the CONMIN and the standard CG method. However, for large problems with inexpensive functions, CG was competitive with L-BFGS. Alekseev et al. (2009) compared the performance of several robust large-scale minimization algorithms for the unconstrained minimization of an ill-posed inverse problem, and they concluded that 1) for the inviscid case, CG-descent method of Hager performed the best, and 2) in the viscous case, the hybrid method emerged as the best performed followed by CG-CONMIN and CGdescent. In an internal tidal model, Chen et al. (2014) concluded that the performance of L-BFGS was better when assimilating all observations, including the observations near open boundaries; however, the gradient descent method will become a good choice if only assimilating the observations far from open boundaries. Therefore in order to determine the best choice of optimization algorithm, performances of GD (Case 1), CONMIN (Case 2), and L-BFGS (Case 3) in Ekman layer model are compared in this subsection.

In the process of parameter optimization, the iteration was executed with enough steps, and the best result was adopted for analysis. The prescribed and inverted VEVCs as well as cost functions versus iteration steps are illustrated in Figs. 1a and 1b. Cost functions and root-mean-square errors (RMSEs) between VEVCs before and after assimilation are shown in Table 1. 
TABLE 1. Cost functions and RMSEs before and after assimilation in Groups 1-8: $K_{1}$ and $K_{2}$ represent the values of normalized cost function with respect to VEVCs before and after assimilation, respectively; and $K_{3}$ and $K_{4}$ are the values of RMSEs between prescribed and estimated VEVCs before and after assimilation, respectively.

\begin{tabular}{|c|c|c|c|c|c|c|}
\hline Group index & Case index & Options & $K_{1}$ & $K_{2}$ & $K_{3}$ & $K_{4}$ \\
\hline \multirow[t]{3}{*}{ Group 1: Algorithms } & Case 1 & GD & $9.8 \times 10^{-2}$ & $3.3 \times 10^{-6}$ & $4.2 \times 10^{-3}$ & $3.2 \times 10^{-4}$ \\
\hline & Case 2 & CONMIN & $9.8 \times 10^{-2}$ & $2.4 \times 10^{-4}$ & $4.2 \times 10^{-3}$ & $4.6 \times 10^{-4}$ \\
\hline & Case 3 & L-BFGS & $9.8 \times 10^{-2}$ & $2.1 \times 10^{-4}$ & $4.2 \times 10^{-3}$ & $4.5 \times 10^{-4}$ \\
\hline \multirow[t]{3}{*}{ Group 2: Initial guess } & Case 4 & 0.0005 & $1.6 \times 10^{-1}$ & $3.7 \times 10^{-6}$ & $4.7 \times 10^{-3}$ & $3.5 \times 10^{-4}$ \\
\hline & Case 5 & 0.005 & $4.1 \times 10^{-2}$ & $1.3 \times 10^{-6}$ & $1.4 \times 10^{-3}$ & $1.1 \times 10^{-4}$ \\
\hline & Case 6 & 0.050 & $1.7 \times 10^{-1}$ & $5.0 \times 10^{-4}$ & $4.5 \times 10^{-2}$ & $4.5 \times 10^{-3}$ \\
\hline \multirow[t]{4}{*}{ Group 3: Distribution } & Case 7 & 1 & $1.0 \times 10^{-1}$ & $3.1 \times 10^{-6}$ & $4.8 \times 10^{-3}$ & $2.5 \times 10^{-4}$ \\
\hline & Case 8 & 2 & $1.2 \times 10^{-1}$ & $2.8 \times 10^{-6}$ & $4.8 \times 10^{-3}$ & $4.0 \times 10^{-4}$ \\
\hline & Case 9 & 3 & $9.8 \times 10^{-2}$ & $2.5 \times 10^{-6}$ & $4.2 \times 10^{-3}$ & $3.0 \times 10^{-4}$ \\
\hline & Case 10 & 4 & $8.6 \times 10^{-2}$ & $4.2 \times 10^{-6}$ & $4.1 \times 10^{-3}$ & $2.9 \times 10^{-4}$ \\
\hline \multirow[t]{4}{*}{ Group 4: Wind condition } & Case 11 & 1 & 1.6 & $5.3 \times 10^{-5}$ & $4.2 \times 10^{-3}$ & $3.2 \times 10^{-4}$ \\
\hline & Case 12 & 2 & $2.4 \times 10^{-1}$ & $6.2 \times 10^{-6}$ & $4.2 \times 10^{-3}$ & $2.4 \times 10^{-4}$ \\
\hline & Case 13 & 3 & $3.6 \times 10^{-1}$ & $2.8 \times 10^{-4}$ & $4.2 \times 10^{-3}$ & $2.3 \times 10^{-3}$ \\
\hline & Case 14 & 4 & $2.0 \times 10^{-1}$ & $2.6 \times 10^{-5}$ & $4.2 \times 10^{-3}$ & $3.3 \times 10^{-4}$ \\
\hline \multirow[t]{4}{*}{ Group 5: BLD } & Case 15 & 40 & $6.3 \times 10^{-1}$ & $1.9 \times 10^{-4}$ & $4.2 \times 10^{-3}$ & $5.2 \times 10^{-4}$ \\
\hline & Case 16 & 80 & $1.7 \times 10^{-1}$ & $9.2 \times 10^{-6}$ & $4.2 \times 10^{-3}$ & $3.5 \times 10^{-4}$ \\
\hline & Case 17 & 120 & $6.1 \times 10^{-2}$ & $1.5 \times 10^{-6}$ & $4.2 \times 10^{-3}$ & $3.0 \times 10^{-4}$ \\
\hline & Case 18 & 160 & $3.0 \times 10^{-2}$ & $4.5 \times 10^{-7}$ & $4.2 \times 10^{-3}$ & $2.9 \times 10^{-4}$ \\
\hline \multirow[t]{3}{*}{ Group 6: Layers } & Case 19 & Odd layers & $6.4 \times 10^{-2}$ & $3.5 \times 10^{-6}$ & $4.2 \times 10^{-3}$ & $3.8 \times 10^{-4}$ \\
\hline & Case 20 & $1-10$ & $9.6 \times 10^{-2}$ & $3.3 \times 10^{-6}$ & $4.2 \times 10^{-3}$ & $3.2 \times 10^{-4}$ \\
\hline & Case 21 & $11-20$ & $1.7 \times 10^{-3}$ & - & $4.2 \times 10^{-3}$ & - \\
\hline \multirow[t]{3}{*}{ Group 7: Data noise } & Case 22 & $2 \%$ & $9.8 \times 10^{-2}$ & $5.8 \times 10^{-5}$ & $4.2 \times 10^{-3}$ & $5.2 \times 10^{-4}$ \\
\hline & Case 23 & $6 \%$ & $9.8 \times 10^{-2}$ & $4.5 \times 10^{-4}$ & $4.2 \times 10^{-3}$ & $7.4 \times 10^{-4}$ \\
\hline & Case 24 & $10 \%$ & $9.9 \times 10^{-2}$ & $1.2 \times 10^{-3}$ & $4.2 \times 10^{-3}$ & $8.7 \times 10^{-4}$ \\
\hline \multirow[t]{4}{*}{ Group 8: Regularization } & Case 25 & $\alpha=1$ & $4.4 \times 10^{-3}$ & $1.2 \times 10^{-8}$ & $4.2 \times 10^{-3}$ & $1.1 \times 10^{-5}$ \\
\hline & Case 26 & $\alpha=10$ & $4.4 \times 10^{-3}$ & $1.2 \times 10^{-8}$ & $4.2 \times 10^{-3}$ & $1.1 \times 10^{-5}$ \\
\hline & Case 27 & $\alpha=100$ & $4.4 \times 10^{-3}$ & $1.2 \times 10^{-8}$ & $4.2 \times 10^{-3}$ & $7.0 \times 10^{-6}$ \\
\hline & Case 28 & $\alpha=1000$ & $4.4 \times 10^{-3}$ & $1.2 \times 10^{-8}$ & $4.2 \times 10^{-3}$ & $2.5 \times 10^{-5}$ \\
\hline \multirow[t]{6}{*}{ Group 9: Balance errors } & Case 29 & $\Delta B=0.01$ & $8.4 \times 10^{-2}$ & $3.0 \times 10^{-6}$ & $4.2 \times 10^{-3}$ & $2.5 \times 10^{-4}$ \\
\hline & Case 30 & $\Delta B=0.1$ & $8.4 \times 10^{-1}$ & $3.5 \times 10^{-5}$ & $4.2 \times 10^{-3}$ & $2.5 \times 10^{-4}$ \\
\hline & Case 31 & $\Delta B=1$ & 8.5 & $3.5 \times 10^{-4}$ & $4.2 \times 10^{-3}$ & $2.5 \times 10^{-4}$ \\
\hline & Case 32 & $\Delta B=0.01$ & $9.9 \times 10^{-2}$ & $4.0 \times 10^{-6}$ & $4.2 \times 10^{-3}$ & $2.4 \times 10^{-4}$ \\
\hline & Case 33 & $\Delta B=0.1$ & $9.9 \times 10^{-1}$ & $3.6 \times 10^{-5}$ & $4.2 \times 10^{-3}$ & $2.4 \times 10^{-4}$ \\
\hline & Case 34 & $\Delta B=1$ & 9.9 & $3.5 \times 10^{-4}$ & $4.2 \times 10^{-3}$ & $2.4 \times 10^{-4}$ \\
\hline
\end{tabular}

In these cases, cost functions are decreased by at least three orders of magnitude and RMSEs by about one order of magnitude. Convergence rates of CONMIN and L-BFGS are much faster than that of the GD. The cost function using CONMIN and L-BFGS reaches the minimum in less than 50 iteration steps, while it is about 350 for GD. However, results in Table 1 demonstrate that inversion results using GD are the closest to the prescribed values among three methods. After assimilation, the cost function using the GD decreases to $3.3 \times 10^{-6}$, which is smaller than that of the other two algorithms. It can be found that the cost function decreases by nearly five orders of magnitude with GD, while less than four orders by using CONMIN and L-BFGS. In addition, Fig. 1a also indicates that inversion results using GD are much smoother than those using L-BFGS and CG. Large fluctuations have been found in both inversion results and cost function when using CONMIN and L-BFGS (Fig. 1b), indicating that CONMIN and L-BFGS is probably not suitable for this problem. The conclusion is similar to those obtained by Chen et al. (2013) and Zhang et al. (2015). Consequently, GD will be selected to estimate the VEVCs in the following groups of experiments.

\section{c. Group 2: Initial guess}

Previous studies have indicated that reasonable initial guess can improve the inversion results and accelerate the convergence (Dattner 2015). Therefore, experiments are designed to test the effect of the initial guess on inverted results in this model. Another purpose is to examine model's ability of tolerating different initial guesses. The initial guesses for Cases $4-6$ are specified as $0.0005,0.005$, 
and 0.05 , respectively. The initial guess of 0.005 equals to the mean value of the prescribed distribution, while the other two options are smaller and larger, respectively. The prescribed and inverted VEVCs as well as curves of cost functions versus iteration steps are illustrated in Figs. 1c and 1d. Cost functions and RMSEs before and after assimilation are shown in Table 1.

It can be found that all inverted VEVCs can converge to the prescribed ones. After assimilation, cost functions and RMSEs are reduced by at least three orders and two orders, respectively. Apparently, results in Case 5 are the best in this group. In Case 5, the cost function and the RMSE decrease to $1.3 \times 10^{-6}$ and $1.1 \times 10^{-4}$, respectively, both of which are smaller than that of Cases 4 and 6. From Fig. 1d, it is easy to find that the fewest iteration steps are used in Case 5, suggesting the highest efficiency of convergence for this case. In addition, Fig. 1c indicates that the fluctuation in Case 5 is much smaller than the other two cases. Results in Group 2 demonstrate the importance of adopting reasonable initial guess in the estimation of time-varying VEVCs in the Ekman layer model. Previous studies have computed VEVCs in realistic ocean, which can provide the basis for initial guess (Yu and O'Brien 1991; Yoshikawa and Endoh 2015).

\section{d. Group 3: Distribution of prescribed VEVCs}

Actual distributions of the VEVCs in the ocean still remain unclear. Consequently, it becomes essential to explore whether the model can deal with different prescribed distributions of VEVCs. In this group, four distributions are designed to test the capability of the model, which are shown as follows:
Case 7: $A(i)=0.003+\ln \left(\frac{i}{48}+1\right) / 600 \mathrm{~m}^{2} \mathrm{~s}^{-1}$;
Case 8: $A(i)=0.003+\ln \left(11-\frac{i}{48}\right) / 600 \mathrm{~m}^{2} \mathrm{~s}^{-1}$;
Case 9: $A(i)=0.005 \times\left[1+0.4 \sin \left(2 \pi \frac{i \Delta t}{4.8 T_{0}}\right)\right] \mathrm{m}^{2} \mathrm{~s}^{-1}$;

$$
\text { Case 10: } A(i)=\left\{\begin{array}{l}
0.003 \mathrm{~m}^{2} \mathrm{~s}^{-1} i=1-48,193-240,385-432 \\
0.005 \mathrm{~m}^{2} \mathrm{~s}^{-1} i=49-96,145-192,241-288,337-384,433-480 \\
0.007 \mathrm{~m}^{2} \mathrm{~s}^{-1} i=97-144,289-336
\end{array}\right.
$$

Inversion results of Group 3 are plotted in Fig. 2. Cost functions and RMSEs before and after assimilation are shown in Table 1. Inversion results in this group validate that the model has the ability to invert VEVCs that are gradually increasing (Case 7), gradually decreasing (Case 8), sinusoidal varying (Case 9), and piecewise-linear varying (Case 10). As can be seen from Table 1, cost functions and RMSEs are decreased by around four orders and one order, respectively. In Figs. 2a and $2 b$, large differences between prescribed and inverted VEVCs always occur at points where prescribed VEVCs is large, which may be due to the small initial guess. Similar results can also be found in Fig. 2c. In Case 10 (Fig. 2d), great differences always appear at discontinuity points. In addition, the results indicate that smoothly changing prescribed VEVCs are easier to be estimated in the model than the sharply changing ones. Moreover, in all the cases the differences are abnormally large at the startup of the time domain due to the small currents at the beginning of simulation introduced by cold start. This phenomenon may arise from sudden variations that break the dynamic balance of the model. Overall, it can be concluded that the Ekman layer model developed in this study has the ability to invert different VEVC distributions.

\section{e. Group 4: Wind condition}

In the real ocean, the wind can be quite different with a wide range. According to Zhang et al. (2009), the mean eddy viscosity under strong winds could increase by about $25 \%$ as compared with that under weak winds in the South China Sea. Consequently, it is worthwhile to examine model's performance under different wind conditions. In this group, four kinds of wind conditions (Fig. 3a) are considered, which are designed as follows:

Case 11: $u_{a}(i)=20 \sin \left(2 \pi \frac{i \Delta t}{T_{0}}\right) \mathrm{m} \mathrm{s}^{-1}$;

Case 12: $u_{a}(i)=10 \mathrm{~m} \mathrm{~s}^{-1}$;

Case 13: $u_{a}(i)=\frac{i \Delta t}{1.2 T_{0}} \mathrm{~m} \mathrm{~s}^{-1}$;

Case 14: $u_{a}(i)=5+\frac{i \Delta t}{2.4 T_{0}} \mathrm{~m} \mathrm{~s}^{-1}$.

Inversion results of Group 4 are plotted in Fig. $3 \mathrm{~b}$. Cost functions and RMSEs before and after assimilation are shown in Table 1. 

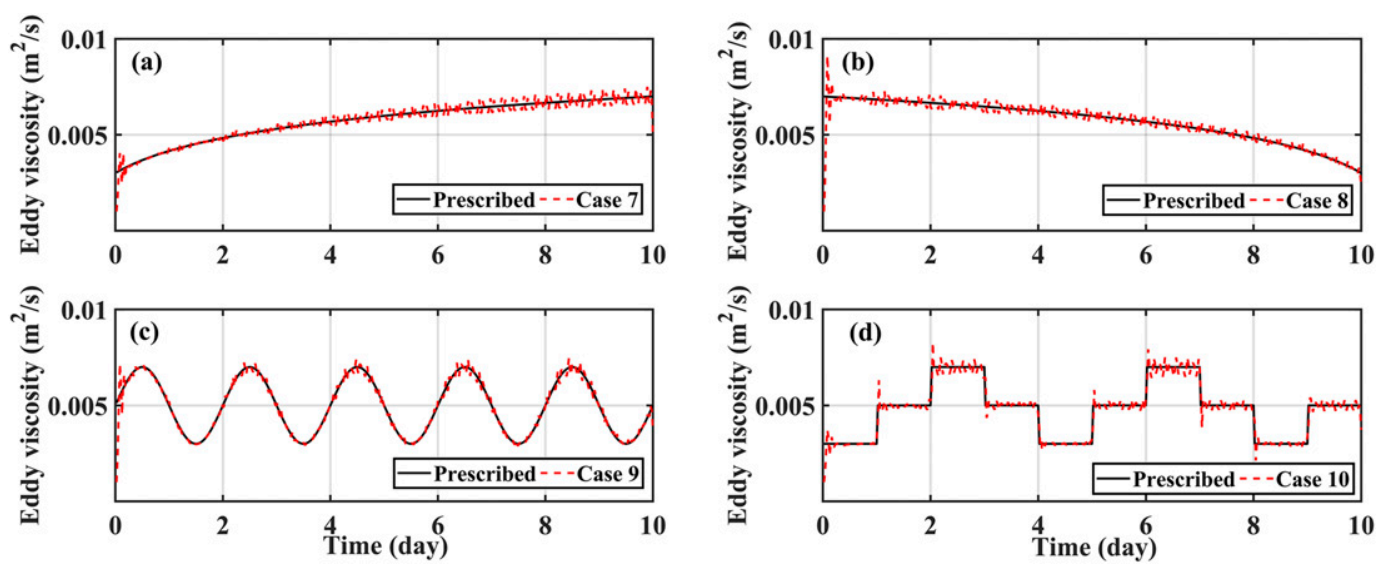

FIG. 2. The prescribed and inverted VEVCs in Group 3: (a) Case 7, (b) Case 8, (c) Case 9, and (d) Case 10.

Reasonable inversion results can be obtained within 400 iterations for Cases 11 and 12. After assimilation, cost functions in Cases 11-14 are decreased by at least three orders of magnitude, and RMSEs are decreased by at least one order, except Case 13. In Case 13 where wind speeds increase from 0 to $10.0 \mathrm{~m} \mathrm{~s}^{-1}$, the inverted VEVCs are apparently unreasonable in the first three days, which can be found in Fig. 3b. As the model is started from a stationary state, when wind speeds are small at the beginning (e.g., the first three days in Case 13), the simulated currents are also small, which results in small gradients and negative influences on the optimization of VEVCs. Comparing Case 13 with Case 14, it is confirmed that small wind speeds at the beginning lead to unreasonable inversion results in the first three days in Case 13. This phenomenon only happens when both wind speed and current velocity are very small for a long time. Given that this situation is uncommon, it is believed that the model can adapt to different wind conditions.

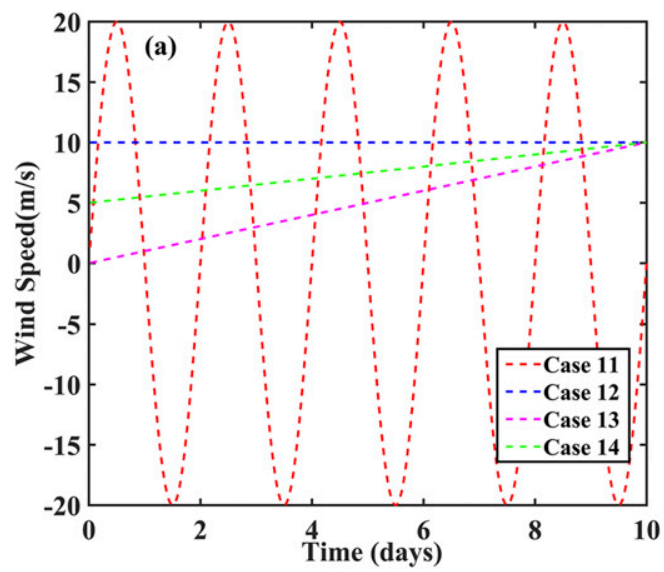

\section{f. Group 5: Boundary layer depth}

The Ekman BLD significantly contributes to the build-up of Ekman currents. Yoshikawa and Endoh (2015) examined the sensitivity of estimated eddy viscosity profile to selected BLD. Jung et al. (2007) emphasized the influence of temporally varying water depth on Ekman motions. In Group 5, four different BLDs are examined, which are 40, 80,120, and $160 \mathrm{~m}$ in Cases 15-18, respectively. The accurate values of BLD are difficult to determine, therefore in this section the BLD in fact is the model covered depth.

Inversion results of Group 5 are plotted in Fig. 4a. Cost functions and RMSEs before and after assimilation are shown in Table 1. Results indicate that all the VEVCs are inverted successfully in Group 5 with different BLDs. When the BLD is larger than $80 \mathrm{~m}$, only a tiny difference exists among the inversion results of Cases 16-18, suggesting that the BLD does not significantly affect the eddy viscosity estimation at

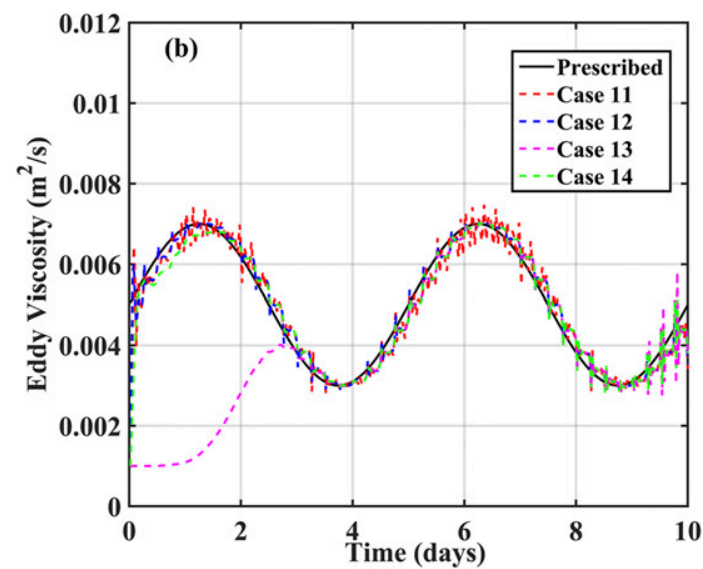

FIG. 3. (a) The wind conditions used in Group 4 and (b) the prescribed and inverted VEVCs in Group 4. 

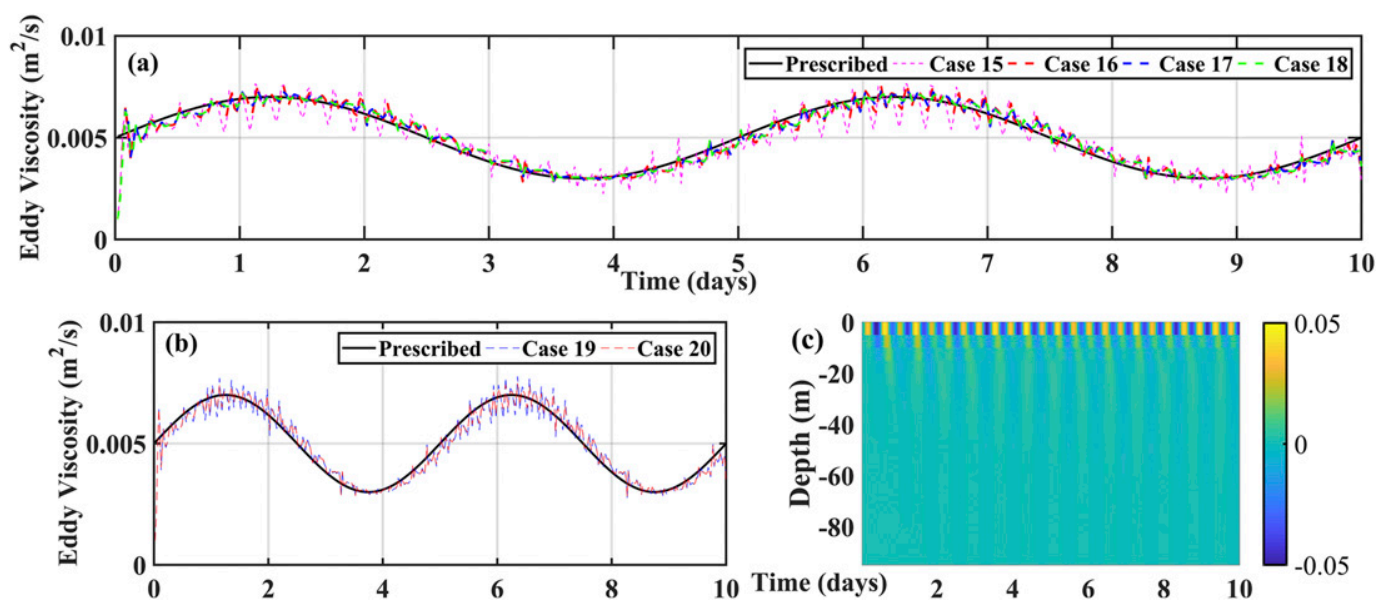

FIG. 4. (a) The prescribed and inverted VEVCs in Group 5, (b) the prescribed and inverted VEVCs in Group 6, and (c) the current observations in Group 6.

these levels. However, when the BLD is decreased from 80 to $40 \mathrm{~m}$ (Case 15), cost functions and RMSEs sharply increase from $9.2 \times 10^{-6}$ to $1.9 \times 10^{-4}$ and from $3.5 \times 10^{-4}$ to $5.2 \times 10^{-4}$, respectively. It seems that there is a critical value of the BLD which can intensely influence the inversion results of time-varying VEVCs. It can be inferred that this critical value should be in close relation with forcing winds (Kukulka and Brunner 2015; Foreman and Emeis 2010). In practice, the critical BLD can be determined by choosing a large value initially and decreasing it until the estimated VEV profile becomes unstable. Results of experiments in this group reveal that the inversion results with thick BLD are slightly better than those with thin BLD under the present wind conditions.

\section{g. Group 6: Vertical levels of available observations}

In the experiments above, simulated velocities at all the layers were used to increase the number of observations in order to restrict the ill-posedness of inverse problem which might be enhanced when time-varying VEVCs is applied. In practice, the data may be not available at some layers because of data missing or data resolution (Yu and O'Brien 1991). In this group, observations from different layers are selected to investigate the effect of the observation level.

Inversion results of Group 6 are plotted in Fig. 4b. Cost functions and RMSEs before and after assimilation are shown in Table 1. In Case 19, observations from odd layers were used; in other words, the vertical sampling interval of this case is $10 \mathrm{~m}$. The result of Case 19 is very close to the result of Case 1, indicating the vertical sampling interval of observations can be enlarged. There is a considerable contrast between the results of Case 20 and Case 21. In Case 20, observations of upper 10 layers are used and the cost function and RMSE are decreased by four orders and one order, respectively. On the contrary, in Case 21 which uses the observations in the bottom 10 layers, the VEVCs have not been optimized. There are two major conclusions of this group. First, this model does not require observations at every layer. Second, the inversion results of VEVCs are much more sensitive to the observations in the upper layers, which is easy to explain that Ekman currents are produced by surface winds and current velocities are larger in upper layers (as shown in Fig. 4c). For the application in real oceans, it is suggested that the sampling resolution in the upper water column should be increased.

\section{h. Group 7: Data noise}

In all the above cases, the observations which are perfect without any noise are directly calculated by the forward model. However, in the real ocean, in situ observations contain noise, which might influence inversion results. Zhang and $\mathrm{Lu}$ (2008) discussed the impact of data noise on inversion results of VEV profiles and open boundary conditions in a three-dimensional tidal model. Their results indicated that when observation errors increased to a certain level, the average difference between inversion results and true values sharply increased.

In this group, in order to evaluate the influence of data noise, observations are assimilated into the model with random errors added as $\left\{\left(1+p r_{d}^{i}\right) u(z),\left(1+p r_{d}^{i}\right) v(z)\right\}$, where $u(z), v(z)$ are currents calculated from the forward model, $r_{d}^{i}$ is a uniform random number lying between -1 and +1 , and $p$ is a factor determining the maximum percentage of errors. The maximum percentages of errors for the three cases are $2 \%$ (Case 22), $6 \%$ (Case 23), and 10\% (Case 24), respectively.

Inversion results of Group 7 are plotted in Figs. 5a and 5b. Cost functions and RMSEs before and after assimilation 

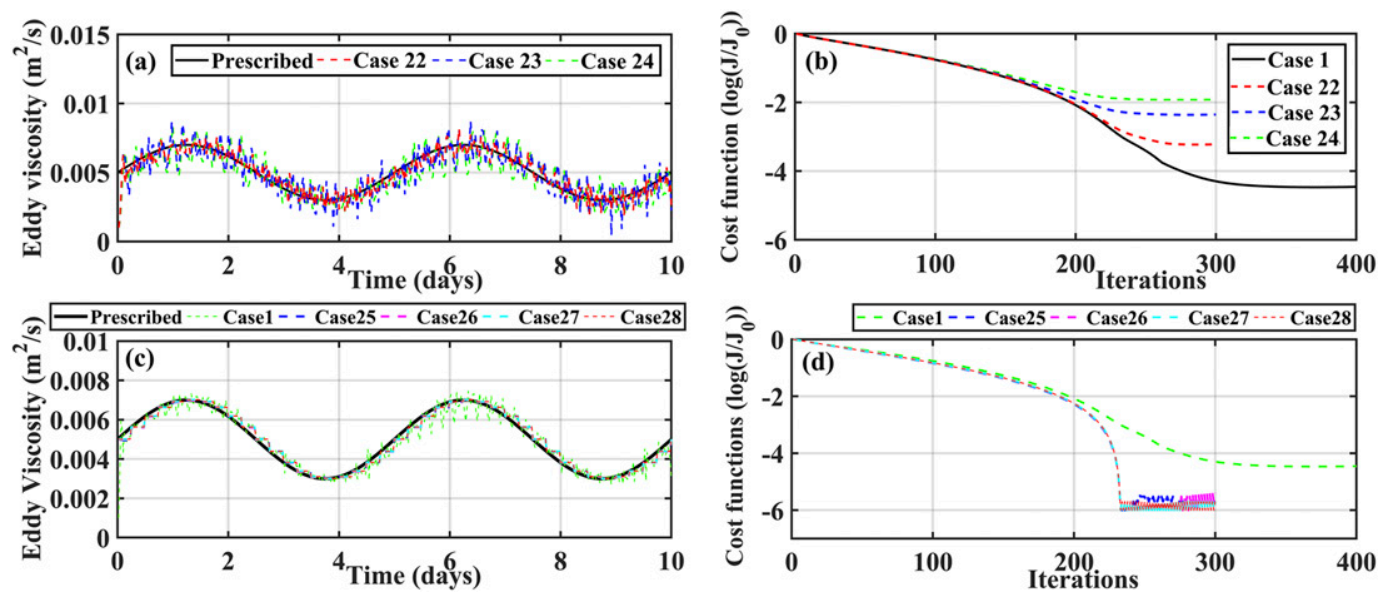

FIG. 5. (a) The prescribed and inverted VEVCs in Group 7, (b) cost functions in Group 7, (c) prescribed and inverted VEVCs in Group 8, and (d) cost functions in Group 8.

are shown in Table 1. In this group, cost functions and RMSEs are clearly increased with the enlargement of random errors. The apparent reduction of RMSEs between prescribed and inverted VEVCs can also be found after assimilation. From Fig. 5a it can be found that the inverted curves in Case 24 oscillate more violently than those in Cases 22 and 23. However, even in Case 24 where the maximum percentage of errors is $10 \%$, the cost function is decreased by nearly two orders (Fig. 5b), and the RMSE is decreased by $80 \%$, indicating that the inversion results are still acceptable. Given that the technologies of measuring ocean currents are under rapid development, the error of velocity measurements from ADCP can be well controlled (Nystrom et al. 2007; De Serio and Mossa 2015), especially after conscious selection and quality control. Therefore, it is believed that the method can sustain data noise to a certain degree.

\section{i. Group 8: Effect of regularization method}

The inverse problems or parameter estimation problems are often ill-posed and beset by instability and nonuniqueness, particularly if one seeks parameters distributed in space and time domain. The regularization method is often used to solve the ill-posedness of inverse problems and has been widely applied in different fields (Tikhonov 1963; Alekseev and Navon 2001; Chen et al. 2014). In regularization method, "stabilizing function" is adopted to restrict admissible solutions to spaces of smooth functions. A smooth cost function is constructed as follows:

$$
\begin{aligned}
J_{\text {regu }} & =J+J_{\text {sta }} \\
J_{\text {sta }} & =\frac{\alpha}{2} \int_{z} \int_{t}(A-\hat{A})^{2} d z d t
\end{aligned}
$$

where $J$ is the cost function defined by Eq. (9); $J_{\text {sta }}$ is the Tikhonov stabilizer; $\alpha$ is the constant regularization parameter; $\hat{A}$ and $A$ are prior and optimized vertical eddy viscosity coefficient, respectively. According to the theory of Lagrangian multiplier method, the corresponding adjoint model and the gradients of smooth cost function with respect to parameters can be obtained as

$$
\begin{aligned}
\frac{\partial J_{\text {regu }}}{\partial A} & =\frac{\partial J}{\partial A}+\alpha(A-\hat{A}) \\
\frac{\partial J}{\partial A} & =-\int_{t} \int_{z}\left(\frac{\partial \lambda}{\partial z} \frac{\partial u}{\partial z}+\frac{\partial \mu}{\partial z} \frac{\partial v}{\partial z}\right) d z d t
\end{aligned}
$$

When $\alpha$ equals to $0, J_{\text {sta }}=0$ and regularization is not performed. If the value of $\alpha$ is very small, the stabilizer function will be also small which will not significantly reduce the ill-posedness of inverse problem; in contrast, if the value of $\alpha$ is too large, the problem will depart far from the original problem and the solution will also be largely distorted. In Group 8, in order to evaluate the influence of $\alpha$ on the estimation results, four experiments are performed in which the values of $\alpha$ are assigned as 0,1 (Case 25), 10 (Case 26), 100 (Case 27), and 1000 (Case 28 ), respectively. As indicated above, $\alpha=0$ is the original experiment without regularization. The results of these experiments are shown in Figs. $5 \mathrm{c}$ and $5 \mathrm{~d}$, and the error statistics are provided in Table 1 . The results demonstrated that the regularization method can indeed improve the estimations results of VEVCs. The cost functions in the experiments without $(\alpha=0)$ and with $(\alpha>0)$ regularization are decreased by around 4.5 and 5.5 orders, respectively (Fig. 5d). The convergence rate with regularization is also faster than that without regularization (Fig. 5d). Also, the fluctuation of estimation VEVCs with regularization is relatively smooth compared with those 

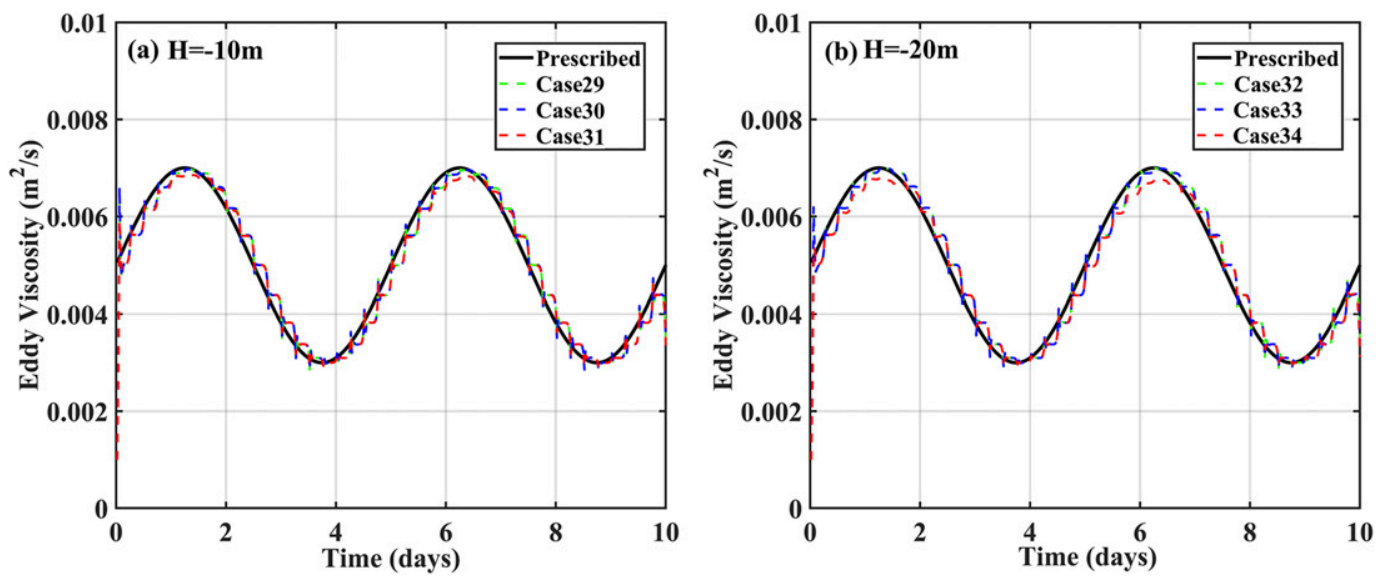

FIG. 6. The prescribed and inverted VEVCs with balance errors in Group 9 at depth (a) $z=10 \mathrm{~m}$ and (b) $z=20 \mathrm{~m}$.

without regularization. Overall, the effect of regularization method in this model is significant; however, it can be further improved by employing more advanced regularization method, such as the wavelets regularization method developed in Alekseev and Navon (2001).

\section{j. Group 9: Sensitivity to balance errors}

With many assumptions in Ekman (1905), the governing equations are not perfect. Following Yoshikawa and Endoh (2015) and Cao et al. (2017), it is assumed that balance errors exist at $z=10 \mathrm{~m}$ and $20 \mathrm{~m}$ with a standard deviation of $\Delta B$, where $\Delta B$ is assigned as $0.01,0.1$, and $1.0 \mathrm{~m} \mathrm{~s}^{-1}$, respectively. Balance errors are added to the governing equations. In Fig. 6 , the prescribed and estimated values of VEVCs with balance errors at depth $z=$ $10 \mathrm{~m}$ (Case 29: $\Delta B=0.01 \mathrm{~m} \mathrm{~s}^{-1}$; Case 30: $\Delta B=0.1 \mathrm{~m} \mathrm{~s}^{-1}$; Case 31: $\Delta B=1.0 \mathrm{~m} \mathrm{~s}^{-1}$ ) and $z=20 \mathrm{~m}$ (Case 32: $\Delta B=$ $0.01 \mathrm{~m} \mathrm{~s}^{-1}$; Case 33: $\Delta B=0.1 \mathrm{~m} \mathrm{~s}^{-1}$; Case 34: $\Delta B=$ $1.0 \mathrm{~m} \mathrm{~s}^{-1}$ ) are plotted. It can be found that when the balance errors are $0.01 \mathrm{~m} \mathrm{~s}^{-1}$ and $0.1 \mathrm{~m} \mathrm{~s}^{-1}$, the estimated VEVCs are quite similar with the prescribed values, but with a small phase shift. When the balance error is $1.0 \mathrm{~m} \mathrm{~s}^{-1}$, the difference becomes relatively large. The phase shift still exists, and there is a difference in the maximum values of VEVCs. However, overall the prescribed time-varying VEVCs can be estimated successfully when balance errors exist in the governing equations, which can partly demonstrate that the present method can tolerate the existence of balance errors due to the imperfection of governing equations.

\section{Practical experiments}

\section{a. Date source}

The idea of time-varying VEVCs has been proved to be effective in identical twin experiments. However, its performance still needs to be testified with real in situ measurements of Ekman currents. To do this, data derived from the Bermuda Testbed Mooring (BTM) are employed in this section; the BTM is a deep-sea mooring (latitude: $31^{\circ} 43^{\prime} \mathrm{N}$, longitude: $64^{\circ} 11^{\prime} \mathrm{W}$ ) located $80 \mathrm{~km}$ southeast of Bermuda, as indicated in Fig. 7a. The water depth is about $4567 \mathrm{~m}$, therefore the bottom friction can be neglected. The sea near Bermuda is usually struck by tropical storms and hurricanes and hence is an ideal area for investigating the upper-ocean's response to wind forcing (Zedler et al. 2002; Black and Dickey 2008).

The BTM program was initiated in 1994 and has provided numerous measurements for testing new instrumentations and performing scientific studies (Jiang et al. 2007). The data selected for this study were collected by BTM deployment 24, and the brief BTM instrumentation diagram used for this deployment (from 14 July 2006 to 1 March 2007) is shown in Fig. 7b.

\section{b. Data processing}

Data from 20 to 30 September 2006, including the wind and water velocity, are used in practical experiments. The Wind observations were measured by the anemorumbometer located at the buoy tower, with sampling interval of five minutes. As the tower was $4.40 \mathrm{~m}$ above the sea surface (Dickey et al. 2001; Black and Dickey 2008), the wind vector was transformed to the standard height $\left(10.00 \mathrm{~m}\right.$ above sea level, $U_{10}$ for short) using a bulk formula (Large and Pond 1981; Zedler et al. 2002; Xu et al. 2017). The original wind speed and $U_{10}$ are plotted in Fig. 8a. From the wind data, one can find an increased stage and a decreased stage of the wind speed are included in selected periods, which is necessary to testify the method. During the selected time stage, the maximum value of wind speed reached $13.66 \mathrm{~m} \mathrm{~s}^{-1}$ at $24-25$ September 2006 and the average wind speed was $5.19 \mathrm{~m} \mathrm{~s}^{-1}$. The current data was measured 
(a)

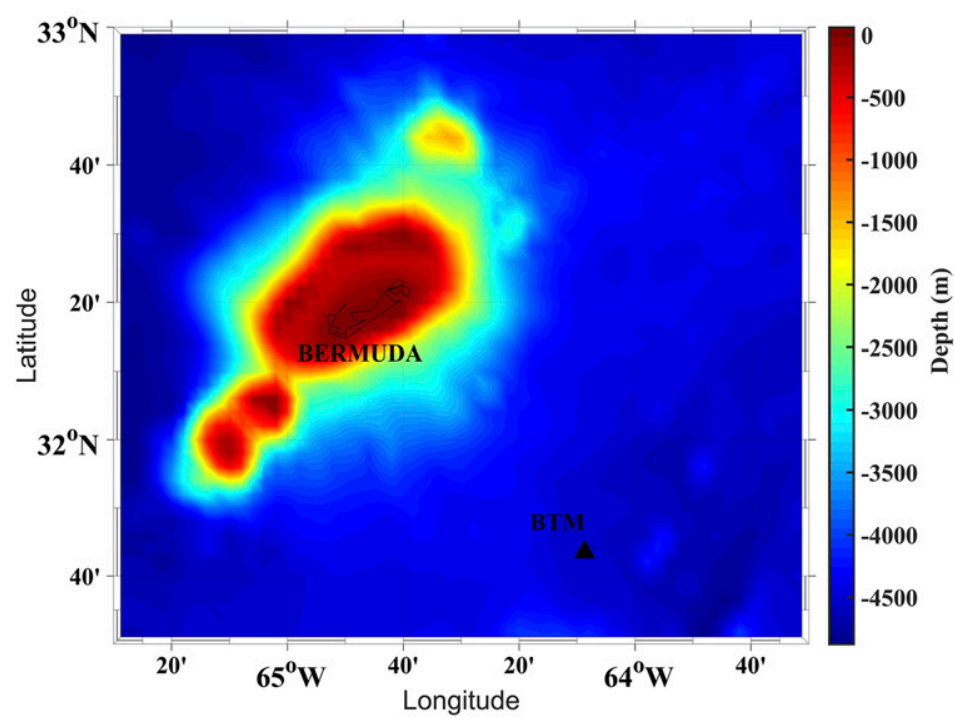

(b)

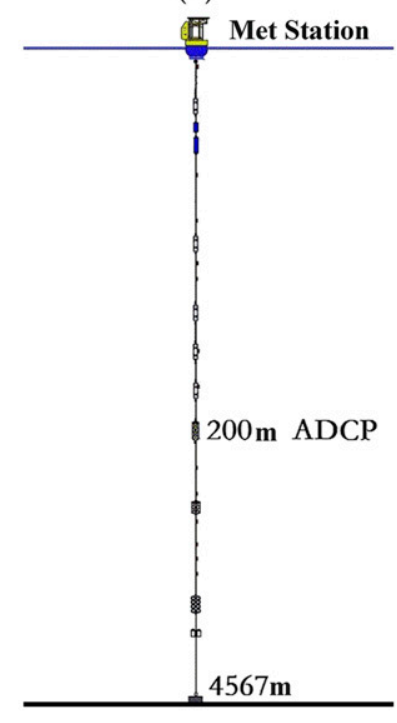

FIG. 7. (a) Topography of study area and the position of BTM and (b) observation instruments diagram of BTM.

by an up-looking ADCP located $200.00 \mathrm{~m}$ below the sea surface, with sampling internal of $15 \mathrm{~min}$ (Dickey et al. 2001; Black and Dickey 2008). With a vertical resolution of $3.00 \mathrm{~m}$, the current profile was measured from 26.52 to $191.52 \mathrm{~m}$ below the sea surface. The time series of the two components of the current speed for the depth of $26.52,50.52$, and $134.52 \mathrm{~m}$ can be found in Figs. 8b-d, respectively.

The measurements of ADCP are the total currents $U_{\text {total }}$, which contain near-inertial signals $U_{\text {ni }}$ and periodical tidal signals $U_{\text {tidal }}$. The near-inertial signals $U_{\text {ni }}$ can be further divided into the wind-generated component
$U_{\mathrm{ek}}$ and the geostrophic component $U_{\mathrm{geo}}$, as indicated in the following equations (Chereskin 1995; Lenn and Chereskin 2009; Roach et al. 2015; Liu et al. 2018):

$$
\left\{\begin{array}{c}
U_{\text {total }}=U_{\text {tidal }}+U_{\mathrm{ni}} \\
U_{\mathrm{ni}}=U_{\mathrm{ek}}+U_{\mathrm{geo}}
\end{array}\right.
$$

This study focuses on the Ekman current $U_{\mathrm{ek}}$, therefore the other two components $U_{\text {tidal }}$ and $U_{\text {geo }}$ should be filtered out. The extraction of Ekman current from in situ current measurements is not easy due to its small magnitude, and many factors can influence
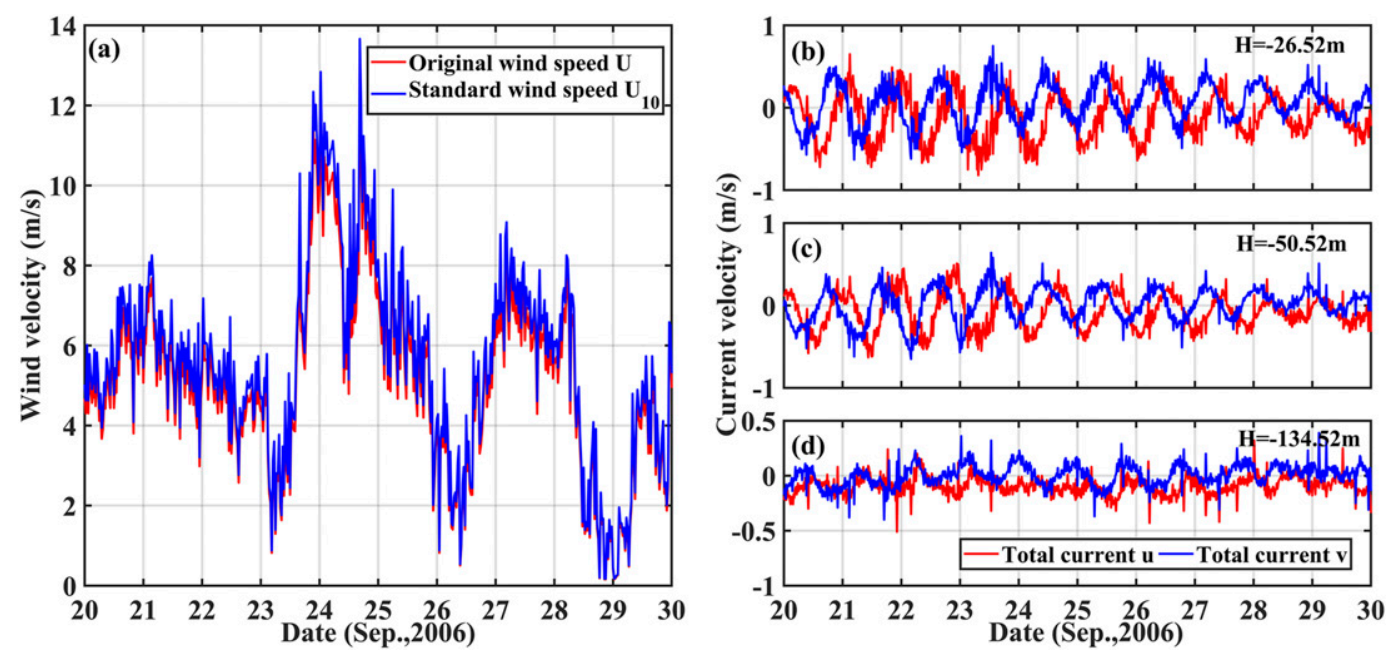

FIG. 8. (a) The time series of the original wind speed $U$ and standard wind speed $U_{10}$ and the time series of total current $u$ and $v$ for (b) $26.52 \mathrm{~m}$, (c) $50.52 \mathrm{~m}$, and (d) $134.52 \mathrm{~m}$. 

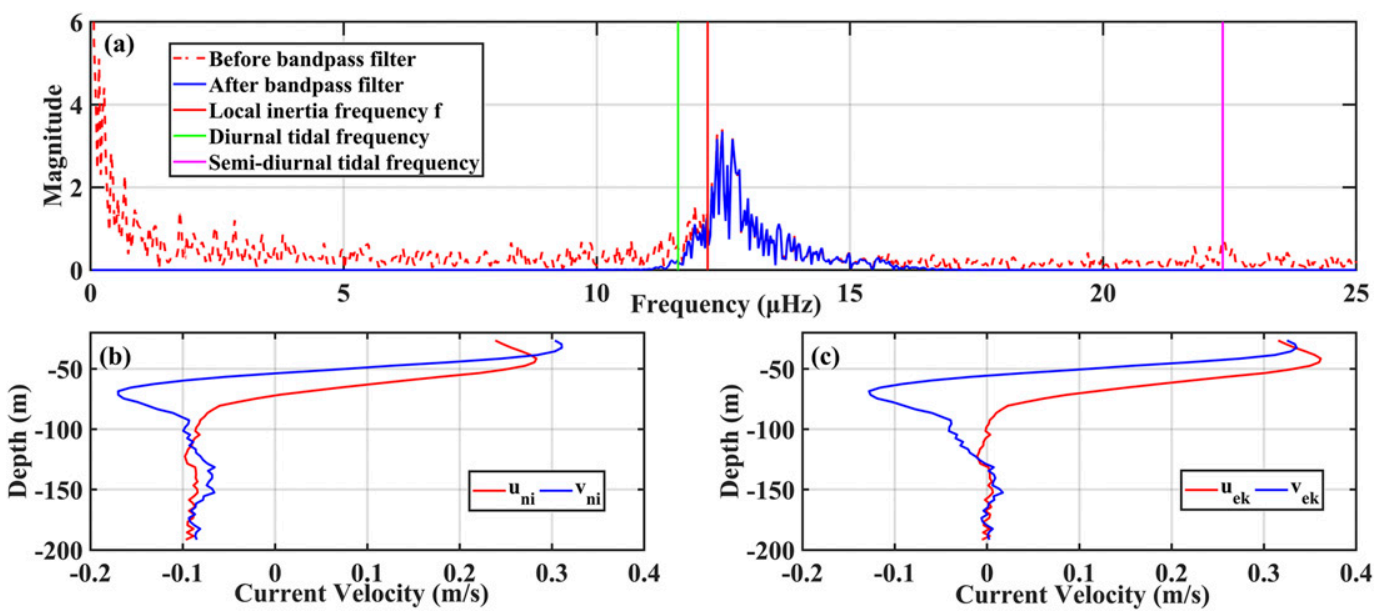

FIG. 9. (a) The spectral analysis results for the currents before and after bandpass filter. Red, cyan, and purple lines indicate the frequency of local inertial, diurnal, and semidiurnal, respectively; (b),(c) the vertical current profiles before and after removing the geostrophic flow at 0000 UTC 20 Sep 2006, respectively.

the extraction results, including the geostrophic current, inertial oscillation, internal wave, and tidal currents, etc. (Polton et al. 2013; Roach et al. 2015). Figure 9a shows the results of the spectral analysis for the time series of current measurements. As shown in this figure, at the study location the frequency of near inertial motion $(f)$ is quite close to the frequency of $\mathrm{P}_{1}$ and $\mathrm{K}_{1}$ tidal constituents. To avoid the disturbance of diurnal tidal signals while retain most of the near inertial signals, the bandpass filter is used in Hui and Xu (2016) and Zhang et al. (2009). If highpass filter is employed, the extraction results of Ekman currents will be influenced by the semidiurnal tidal signals $\left(\mathrm{M}_{2}, \mathrm{~S}_{2}\right.$, and $\mathrm{K}_{2}$ etc.). Another method of eliminating tidal signals is to subtract the tidal signals calculated from tidal current harmonic constants. However, the precondition of this method is solid results of tidal current harmonic constants are available which must be calculated from long-term in situ current measurements. The harmonic constants deduced from short-term current measurements will be questioned due to low accuracy (Foreman and Henry 1989).

As in Hui and Xu (2016) and Zhang et al. (2009), in this paper the tidal signals are removed by using the Hilbert transform bandpass filter. According to Fig. 9a, the bandpass frequency was selected to be [0.96 $f 1.3 f]$, where $f$ is the local inertial frequency (Hui and $\mathrm{Xu} 2016$ ). The current field after bandpass filter can be found in Fig. 10. The spectral analysis results of filtered currents are also plotted in Fig. 9a, and one can find most of diurnal and semidiurnal tidal signals have been removed.

In the calculation of Ekman current velocity, the assumption of constant $U_{\text {geo }}$ below a certain water depth can introduce errors in the separation between Ekman current and geostrophic current (Polton et al. 2013; Phillips and Bindoff 2014). For example, the assumption of a constant geostrophic velocity within the mixed layer is not supported by observations undertaken during the Southern Ocean Fine-Structure (SOFINE) experiment (Phillips and Bindoff 2014). A detailed description about the separation between these two components can be found in Roach et al. (2015). As a result, the method in Roach et al. (2015) was adopted, where the geostrophic current was assumed to be a sum of a constant reference velocity $U_{\text {deep }}$, and a component arising from a constant geostrophic shear $\left(d U_{\text {geo }} / d z\right) z$ :

$$
U_{\text {geo }}(t)=U_{\text {deep }}(t)+\frac{d U_{\text {geo }}(t)}{d z} z
$$

To determine the values of $U_{\text {deep }}$ and $\left[d U_{\text {geo }}(t) / d z\right] z$, the vertical current profile at the initial time after the bandpass filter was plotted in Fig. 9b. One can find the variations of current speed is quite small below $161.52 \mathrm{~m}$, therefore $U_{\text {deep }}$ is taken as the average value of $U_{\text {ni }}$ below this depth. According to Ralph and Niiler (1999), the Ekman depth $D_{E}$ is approximately estimated as $134.52 \mathrm{~m}$ by using

$$
D_{E}=\frac{7.12}{\sqrt{\sin |\varphi|}} U_{10},
$$

where $\varphi$ is the latitude of BTM, $U_{10}$ is taken as the maximum wind speed with a value of $13.66 \mathrm{~m} \mathrm{~s}^{-1}$ for the selected time period. $d U_{\text {geo }}(t) / d z$ is then obtained by computing the average value of the vertical current shear for the depth 134.52 to $161.52 \mathrm{~m}$. A snapshot of 


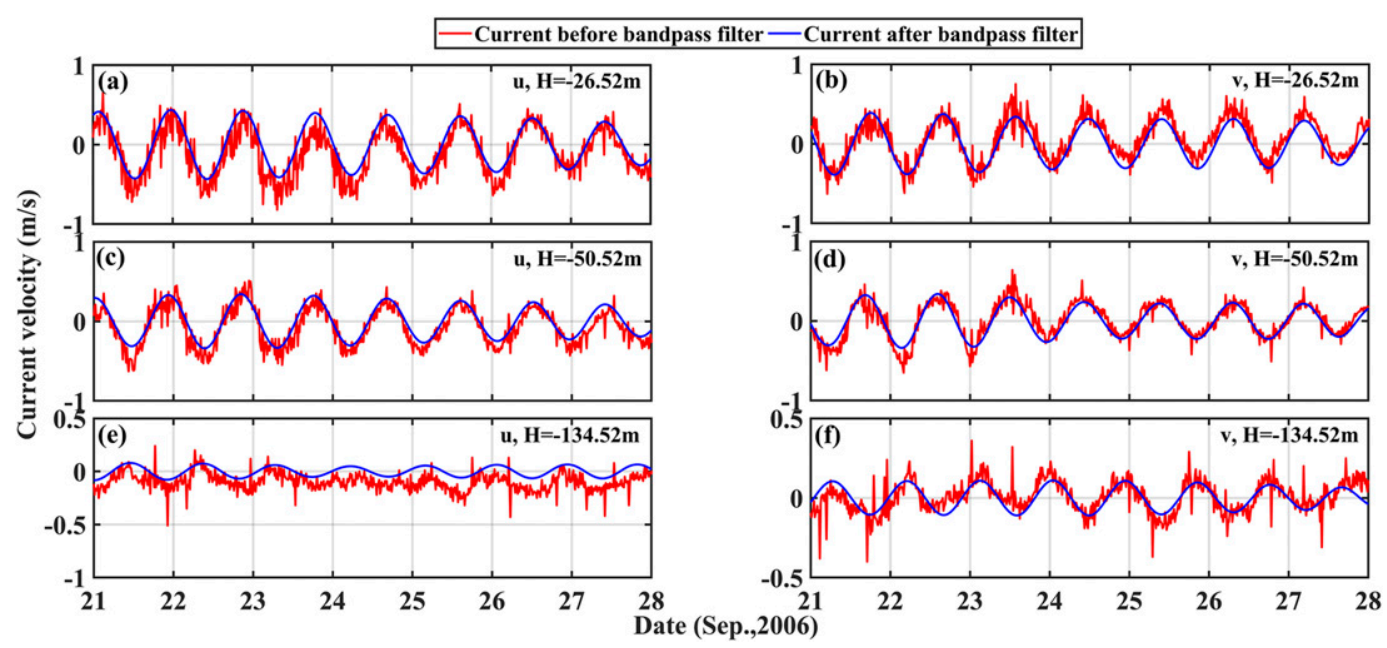

FIG. 10. Time series of currents before and after bandpass filter: time series of (left) eastward current velocity and (right) northward current velocity at (a),(b) $26.52 \mathrm{~m}$; (c),(d) $50.52 \mathrm{~m}$; and (e),(f) $134.52 \mathrm{~m}$.

vertical profile of Ekman current at 0000 UTC 20 September 2006 after removing the geostrophic component can be found in Fig. 9c. The values of Ekman currents at different water depth can be obtained by linearly interpolating the values at sampling depth.

\section{c. Inverse strategy}

To compare different methodologies of VEVCs, in this section three strategies are employed. The first one (Case 35) assumes the VEVC is a constant which neither varies with time nor with space (depth). The second one (Case 36) assumes that the VEVCs is depth dependent, and the last one (Case 37) is the time-varying VEVC described in this paper. According to Peng and Li (2015), the wind drag coefficient $C_{D}$ is calculated by

$$
C_{D}(i)=-a\left[\left|u_{10}(i)\right|-33.0\right]^{2}+c,
$$

where $a=2.0 \times 10^{-6}, c=2.34 \times 10^{-3}, i=1-481$. In Case 35 , the constant value of $A$ is taken as $0.008 \mathrm{~m}^{2} \mathrm{~s}^{-1}$, and the initial values for Cases 36 and 37 are also both set to this value. The initial state of the model is obtained by using the BTM current observations at 0000 UTC 20 September 2006, and the model is forced by using the BTM observed wind fields. The time step of the model is set to $0.5 \mathrm{~h}$. The first day (0000 UTC 20 September to 0000 UTC 21 September) is used for model spinup. The model is integrated forwardly in the following seven days to generate modeling Ekman current fields, and the adjoint model is integrated backwardly to calculate the adjoint variables, based on which the VEVCs can be optimized and inverted as described in section 2. The other settings are the same as those in section 3 .

\section{d. Results analysis}

The modeled and observed Ekman currents of 26.52, 50.52, 65.52, and $98.52 \mathrm{~m}$ in Cases $35-37$ are plotted in Fig. 11. For the modeled currents above $65.52 \mathrm{~m}$, both the amplitude and phase coincide with observations fairy well, especially the results of Cases 36 and 37. The results of Case 36 follow Case 37 with tiny difference, and the results of Case 35 are the worst among three cases. The tiny difference between Cases 36 and 37 demonstrate that the assumptions of depth dependence and time dependence are equally important for VEVCs. Different from current fields above $65.52 \mathrm{~m}$, the modeling results below $65.52 \mathrm{~m}$ have shown relatively large discrepancies from observations, existing in both the amplitude and phase. The reasons might be that 1) the influence of wind under this depth is too small, resulting in decreased values of Ekman currents, and 2) the observation errors and effects of other dynamics might have distorted the measurements.

The decrease of normalized cost function versus the iteration steps can also demonstrate the effectiveness of the method, as shown in Fig. 12a. All the normalized cost function in three practical experiments have been decreased significantly from 1.0 to around $0.3-0.4$, which indicates that the adjoint method is effective in reducing the data misfit between observed and modeled Ekman currents. The minimum value of cost function has been reached in Case 37, indicating that the methodology of time-varying VEVCs is better than the other two assumptions. Besides, the variations of cost function versus iteration steps in Case 37 are the smoothest; contrarily, the cost functions in Cases 35 and 36 fluctuate significantly after a certain iteration step. The time series 


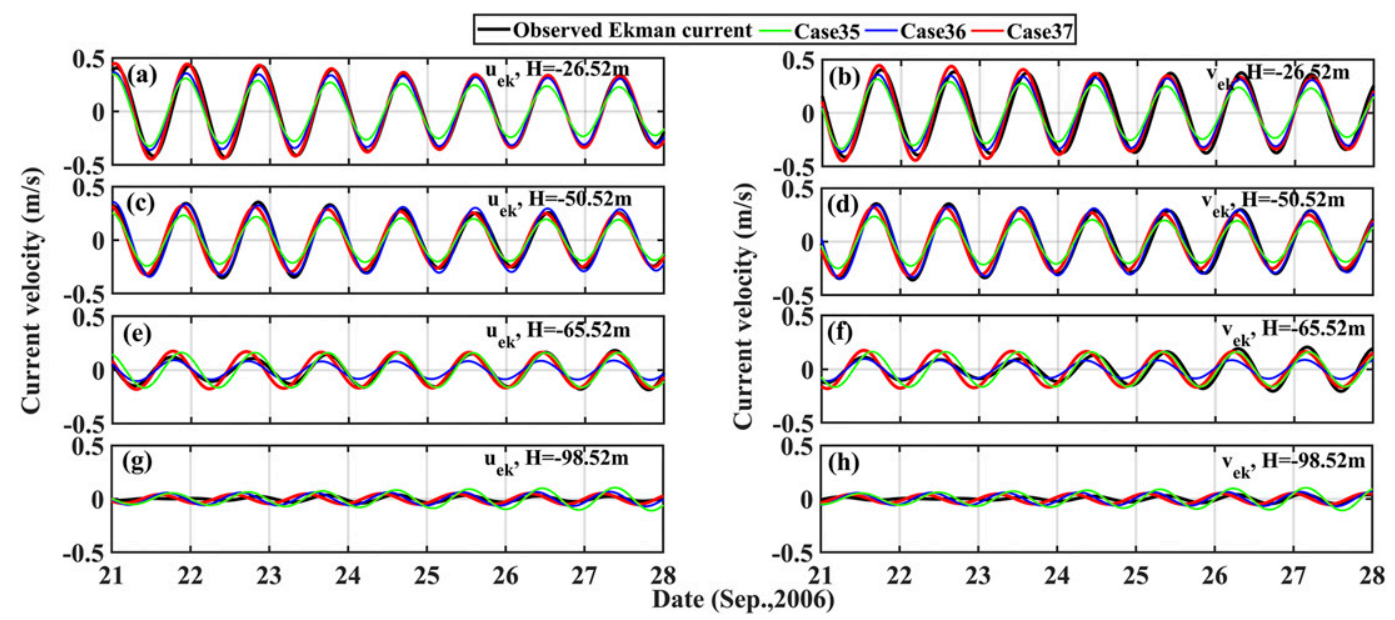

FIG. 11. The observed and modeled (left) eastward and (right) northward Ekman current time series of (a),(b) $26.52 \mathrm{~m}$; (c),(d) $50.52 \mathrm{~m}$; (e),(f) $65.52 \mathrm{~m}$, and (g),(h) $98.52 \mathrm{~m}$ in Cases 35-37.

of VEVCs inverted in Case 37 is plotted in Fig. 12b. The average and maximum vales of the estimated VEVCs (unscaled) are 0.0021 and $0.0049 \mathrm{~m}^{2} \mathrm{~s}^{-1}$, respectively. The tendency for the time variations of VEVCs closely related to the variations of wind speed. For the periods of 24-26 September 2006, the wind speed increased from a minimum value of smaller than $2.00 \mathrm{~m} \mathrm{~s}^{-1}$ to the maximum value of $13.66 \mathrm{~m} \mathrm{~s}^{-1}$, and then decreased to below $1.00 \mathrm{~m} \mathrm{~s}^{-1}$. The similar variations of estimated VEVCs, with a phase shift, can also be found in Fig. 12b. There are two peak values of VEVCs and happened during 24-25 September 2006 and 2627 September 2006, respectively, which agree well with the peak values of wind speed. The estimation results are in accordance with the traditional knowledge that the VEVCs can be highly influenced by the wind fields. In this paper the depth variance of VEVCs is not considered, but the magnitudes of estimation results are comparable with the results of $\mathrm{Yu}$ and
O'Brien (1991) and Price et al. (1987). In their work which studied the estimation of depth-dependent VEVCs in the Sargasso Sea $\left(\sim 34^{\circ} \mathrm{N}\right)$, the estimated VEVCs were $0.0029 \mathrm{~m}^{2} \mathrm{~s}^{-1}$ for the surface layer, and decreased downwardly. The modeled and observed Ekman currents in Cases 37 at depth 26.52, $32.52,38.52,44.52,50.52$, and $56.52 \mathrm{~m}$ at 0000 UTC 20 September 2006 can be found in Fig. 13. Both the magnitude and direction of currents agree well with each other, indicating that the combination of timevarying VEVC and adjoint method can generate reasonable Ekman currents.

\section{e. Sensitivities to formulae of wind drag coefficients}

In this section, numerical experiments are carried out to study the impact of different formulae of wind drag coefficient on the estimation of VEVCs. Including Peng and $\mathrm{Li}$ (2015), four formulas of wind drag coefficients are compared. The other three are given as follows:

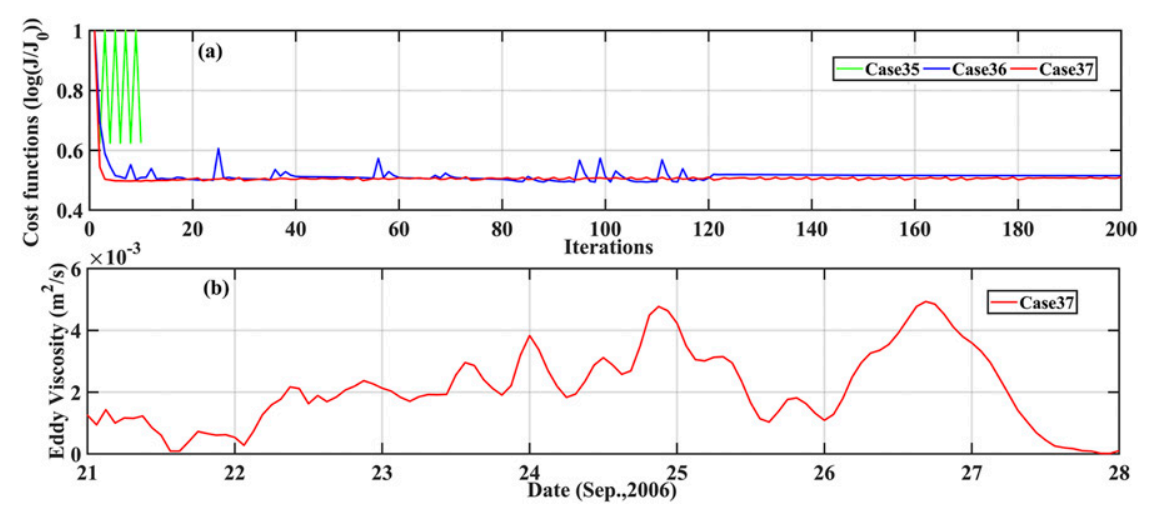

FIG. 12. (a) The values of normalized cost function with respect the iterations in Cases 35-37.

(b) The time series of VEVCs (unscaled) estimated in Case 37. 


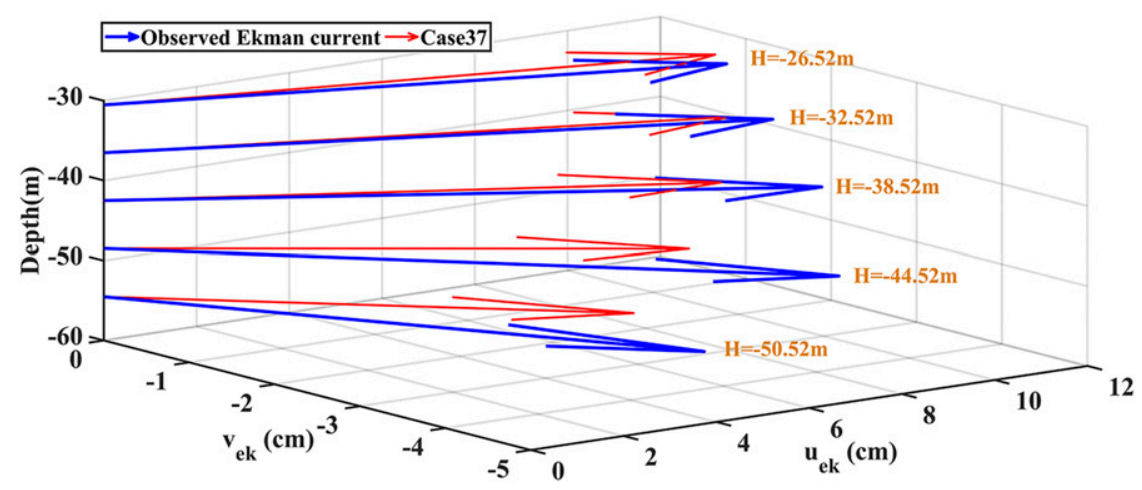

FIG. 13. The snapshot of modeled and observed Ekman currents in Cases 37 at depths of 26.52, 32.52, 38.52, 44.52, 50.52, and 56.52 $\mathrm{m}$ at 0000 UTC 20 Sep 2006.

Case 38 with formula in Edson et al. (2013):

$$
C_{D}(t)=\left[0.062-0.28 /\left|u_{10}(t)\right|\right]^{2} .
$$

Case 39 with formula in Zijlema et al. (2012):

$$
C_{D}(t)=\left[0.55+2.97 / 31.5-1.49\left|u_{10}(t)\right|^{2} / 31.5^{2}\right] \times 10^{-3} \text {. }
$$

Case 40 with formula in Large and Yeager (2009):

$$
\begin{aligned}
C_{D}(t)= & 2.70 \times 10^{-3} /\left|u_{10}(t)\right|+1.42 \times 10^{-4} \\
& +7.64 \times 10^{-5}-3.14807 \times 10^{-13}\left|u_{10}(t)\right|^{13} .
\end{aligned}
$$

The estimation results of VEVCs with these different forms of wind drag coefficients can be found in Fig. 14. It can be found that, either the decrease of cost function, or the estimation results of VEVCs, both show quite similar patterns among the four experiments. The average and maximum values of estimated VEVCs in these four cases are also very close. Only tiny differences exist, especially during the periods when the wind was weak, which happened in the first three days in Fig. 14b. After that, during the period with high wind speed, the estimated VEVCs with four different formulae of wind drag coefficients are of little difference. It indicates that the selection of wind drag coefficient formulae will not significantly influence the estimation of VEVCs in this paper. However, it should be noted that, during the study period, the wind speed is not as large as that in typhoon or hurricane; therefore, the above conclusion might be only correct for this type of wind field. For quite high wind speed, such as during a typhoon or hurricane, the selection of wind drag coefficient formulae might have a significant influence on the estimation of VEVCs, which will be studied in the future.

\section{Discussion}

\section{a. Feasibility of typical Ekman model}

The typical Ekman theory, developed in Ekman (1905), describe the steady spiraling velocity profiles in boundary layers, in which the constant of proportionality

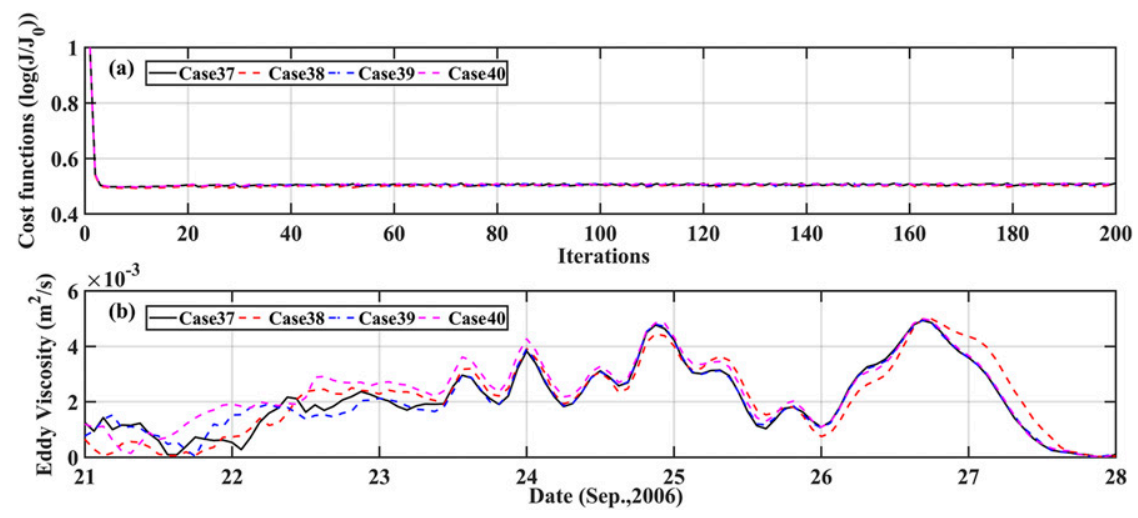

FIG. 14. The prescribed and estimated VEVCs with different wind drag coefficient formulae:

(a) cost functions vs iteration steps and (b) estimated VEVCs (unscaled). 
between the stress and shear, called the turbulent eddy viscosity, is applied. Whether the typical Ekman theory can be applied in the real ocean has been studied continuously (Price et al. 1987; Chereskin 1995; Elipot and Gille 2009; Lenn and Chereskin 2009; Polton et al. 2013; Roach et al. 2015). These studies found the classical constant viscosity was inadequate and should be improved. However, Ekman currents are difficult to be observed. This is primarily because they are small signals that are easily masked by ocean variability and cannot readily be separated from the geostrophic component. A comprehensive description about this can be found in Roach et al. (2015). Their results on detecting and characterizing the Ekman currents in the Southern Ocean indicate that the constant viscosity Ekman model can offer a reasonable description of momentum mixing into the upper ocean in the Antarctic Circumpolar Current north of Kerguelen. Polton et al. (2013) performed an interesting work to discuss whether ADCP observations in the Drake Passage can match Ekman's classic theory, by seeking a suitable value of eddy viscosity, and their results are positive. They found that values of VEVC in the range of 0.08 $0.12 \mathrm{~m}^{2} \mathrm{~s}^{-1}$ can reconcile their observations with the classic Ekman theory. From previous works, two conclusions can be inferred: 1) the classical Ekman theory can be used to figure out the upper-ocean response under wind forcing; however, in order to do this, the eddy viscosity needs adjusting, and 2) the eddy viscosity is not a constant value and should be space dependent or time dependent; in other words, it can be seemed as observation dependent for most cases, which significantly increases the difficulty of determining the values.

This paper focuses on the time-varying eddy viscosity to improve the Ekman layer model by assimilating in situ observations with adjoint method. The eddy viscosity in our method is thus observation dependent. The advantage of this methodology is that the determination of eddy viscosity can be converted to the process of data-model consistency, with the help of optimization methods and idea of adjoint parameter estimation (Navon 1998). As shown in Fig. 13, the snapshot of modeled and observed Ekman currents indicate they agree well with each other, including both the magnitude and direction of currents, which demonstrate that typical Ekman theory can be applicable by adjusting the VEVCs (Polton et al. 2013).

\section{b. Imperfection of balance equations}

Governing equations of Ekman layer model are given in Eqs. (1). In the primitive work of Ekman (1905), combined with the boundary conditions, the solutions are given as

$$
\left\{\begin{array}{l}
u=V_{0} \exp (\gamma z) \cos (\pi / 4+a z) \\
v=V_{0} \exp (\gamma z) \sin (\pi / 4+a z)
\end{array}\right.
$$

where $V_{0}$ is the velocity of the current at the sea surface, and has the expressions

$$
V_{0}=\frac{T}{\sqrt{\rho_{w}^{2} f A}}, \quad \gamma=\sqrt{\frac{f}{2 A}} .
$$

Solutions (43) are suitable for typical Ekman theory: homogeneous density, no stratification, constant VEVC, deep water, etc. However, the stratification can influence the dynamics in Ekman layers by restraining the turbulence and reducing the efficiency of wind energy input to ocean interior. According to the parameterization method in Pacanowski and Philander (1981), the effect of stratification can be introduced by improving constant VEV as follows:

$$
A=\frac{v_{0}}{\left(1+\alpha R_{i}\right)^{2}}+v_{b}
$$

where $\quad \alpha=5, \quad v_{0}=0.01 \mathrm{~m}^{2} \mathrm{~s}^{-1}, \quad v_{0}=0.001 \mathrm{~m}^{2} \mathrm{~s}^{-1}$, and $R_{i}$ is the Richardson number, which can be expressed by the vertical density gradient and velocity shear, as follows:

$$
R_{i}=\frac{\left(d \rho_{w} / d z\right) g / \rho_{w}}{(\partial U / \partial z)^{2}+(\partial V / \partial z)^{2}},
$$

where $U$ and $V$ are the two components of current.

From (43)-(45) we can find that stronger stratification leads to larger Richardson number, which then results in smaller vertical eddy viscosity, and vice versa. Therefore, the solutions (43) are also applicable if VEV is improved by introducing the effect of stratification, for example with the method of (44) and (45). In this work, although we employed the typical Ekman model, the VEVC is not a constant but estimated by assimilating in situ observations. If we take the ocean as a complex system which is driven by different forces, the in situ observations are actually the outputs of this system. The observations (output) are generated by various factors, including stratification, wind, horizontal pressure gradient, etc. As a result, the VEVCs estimated by assimilating in situ observations are in fact the implicit functions of these factors. It is also an advantage of this methodology, which is based on the theory of inverse problem and parameter estimation, and can be taken as an improved method compared with the traditional parameterization.

Nevertheless, with many assumptions in Ekman (1905), the governing equations are not perfect. The 


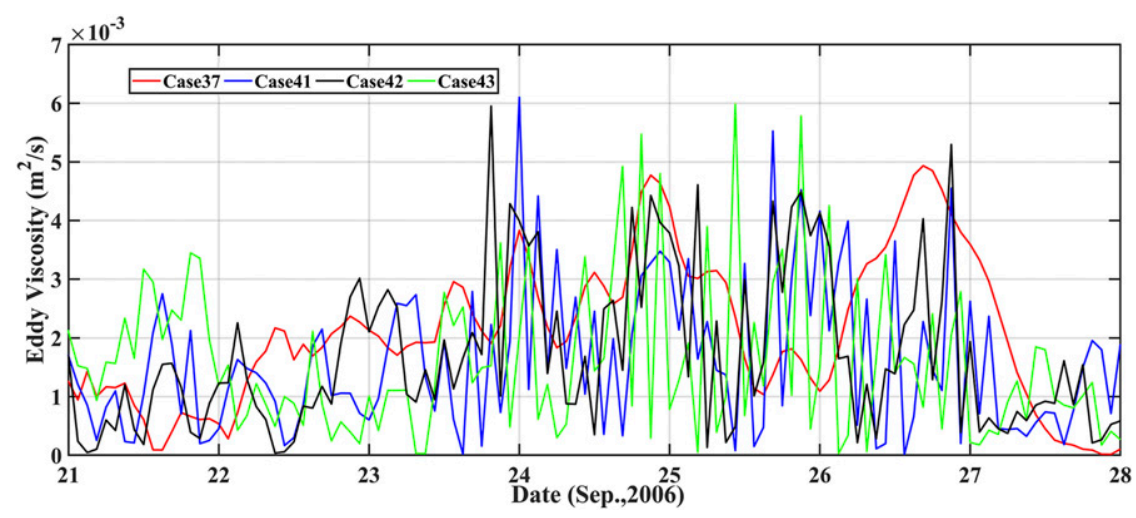

FIG. 15. The time series of estimated VEVCs in Case 37 (without balance error) and Cases $41-$ 43 (with balance error).

classical Ekman model is not totally successful as some observational evidence does not directly support it (Price and Sundermeyer 1999; Lewis and Belcher 2004; Polton et al. 2005; Wu and Liu 2008; Song 2009). As studied in Yoshikawa and Endoh (2015) and Cao et al. (2017), because of the existence of advection, pressure gradient, stratification and other terms in the real motion, there would be errors in the Ekman balance equations. For the present method, the addition of these terms will make the adjoint model too complex, because the baroclinic field has to be solved with these terms, which is generally involved in complex general circulation models, such as ROMS, MITGCM, and FVCOM, etc. Besides, the complexity and difficulty of parameter estimation will also be significantly increased. Therefore, instead of involving these terms, the sensitivity of the present method to Ekman balance errors is investigated in this section. Following Yoshikawa and Endoh (2015) and Cao et al. (2017), as in section $3 \mathrm{j}$, it is assumed that balance errors $(\Delta B)$ exist at governing equations when carrying out practical experiments. In Case $41, \Delta B=$ $0.001 \mathrm{~m} \mathrm{~s}^{-1}$ is assigned at $z=-11.52 \mathrm{~m}$; In Case 42, $\Delta B=0.001 \mathrm{~m} \mathrm{~s}^{-1}$ is assigned at $z=-21.52 \mathrm{~m}$; and in Case $43, \Delta B=0.001 \mathrm{~m} \mathrm{~s}^{-1}$ is assigned at both $z=-11.52 \mathrm{~m}$ and $z=-21.52 \mathrm{~m}$. In Fig. 15, the results of experiments without balance errors (Case 37) and with balance errors (Cases 41-43) are shown. We can find that, in experiments with balance errors, especially Case 41 and Case 42, the estimation results of VEVC can better coincide with the wind fields (shown in Fig. 8a). It demonstrates that the unresolved mechanisms can introduce estimation errors of VEVC. However, the overall pattern in these cases are quite similar, especially during the phases that wind speed varied significantly, indicating that the estimation results can reflect the variation of VEVC to some degree.

\section{c. Deficiencies and future work}

In this paper, the wind speed is not very high, which can consequently reduce the response of the upper ocean to the atmosphere forcing. For very high wind speed, such as the period of typhoons or hurricanes, the question will be quite different, which is a big challenge in oceanography. The enhanced mixing, the bubbles, the rough sea surface, and the Langmuir circulation will all influence the structures of VEVCs and Ekman current, both in space and time domains. In this situation, the typical Ekman theory might not be able to figure out the response of the upper ocean to extreme events. In the future work, we will focus on the improvement of the adjoint Ekman model to represent the effects of strong winds. A possible method is to modify the Ekman model by including the wave effects (Lewis and Belcher 2004; Polton et al. 2005; Liu et al. 2007; Wu and Liu 2008; Hui and $\mathrm{Xu}$ 2016). By taking the wave-induced Coriolis-Stokes forcing into account, the impact of waves (primarily the Stokes drift) on ocean surface currents is investigated and the wave-modified currents are formed in Hui and $\mathrm{Xu}$ (2016). Their results with the Stokes drift can better adapt to the in situ Lagrangian drifter currents, especially in the Southern Ocean region where the wind is strong. The reason is that the magnitude of the Stokes drift or the wavemodified term is directly related with the strength of the surface wind; therefore, the wave-modified term is especially large in strong wind areas or under strong wind conditions. Overall, by considering the wave effects in the present adjoint Ekman model, the methodology might be applicable in strong wind conditions.

For the real ocean, the parameter space can be as complicated as four-dimensional and case/observation 
dependent due to the turbulence, and that is why the parameterization is too difficult to be perfect. It will be much more reasonable to suppose the VEVCs to be vertically and temporally varying. However, for the inverse problem, it is difficult to realize the fourdimensional parameters, depending on the quality and quantity of in situ observations, including the horizontal, vertical and temporal resolutions, noise level, etc. Therefore, an eclectic method is focusing on a certain dimension, depth dependent or time dependent. Also, it will not be a copy of the present problem by adding one dimension, because new methods of inverse problem might be necessary to constrain the ill-posedness, such as advanced regularization method (Alekseev and Navon 2001). The vertical and temporal VEVCs will be considered simultaneously in the future work after carrying out adaptive observing and developing suitable estimation strategy.

\section{Conclusions}

Except some ideal case for the purpose of simplicity, the ocean is a time-varying system and the parameters decided by ocean dynamics should also be time varying. Based on an Ekman layer model, the estimation of timevarying VEVCs is studied with adjoint assimilation method in this paper.

A series of ideal experiments are carried out to examine different influencing factors on estimation results, including optimization algorithm, initial guess, prescribed distributions, wind conditions, BLD, observation level, data noise, regularization method, and balance error. Three algorithms (GD, CONMIN, and L-BFGS) are discussed in Group 1. CONMIN and L-BFGS have a large advantage of the convergence rate; however, GD shows better performance in the final estimation results, which is consequently adopted in the following experiments. In Group 2, three different initial guesses are tested. Although results using different initial guesses all can converge to prescribed values, the initial guesses should be as reasonable as possible to increase the efficiency. The ability of the present method of estimating different prescribed distributions is examined in Group 3. All the results are acceptable, indicating that the model is able to invert different distributions of VEVCs. In Group 4, the influence of different kinds of wind conditions is examined. Results demonstrate that the model is applicable to different wind conditions including sinusoidal, constant and linear changing wind speeds. However, if both wind speeds and Ekman velocities are too small simultaneously, the inversion results will dramatically be poor. The response of the model to BLD is studied in Group 5. Results reveal that inversion results with thick BLD are better than those with thin BLD. In Group 6, different observation levels are selected and compared. The results are more sensitive to observations in the upper layers than in the lower layers. Group 7 discusses the influence of data noise in observations. The inversion results are acceptable when data noise exists, indicating the method can sustain noise to a certain degree. Since technologies have been undergoing a rapid development, the accuracy of current observations can ensure the accuracy of the VEVC estimation. In Group 8, the effect of regularization method is studied and the results are positive that it can improve the estimation results. The results of Group 9 indicate that the present method can tolerate the existence of balance errors due to the imperfection of governing equations.

The methodology is further validated in practical experiments where Ekman currents are derived from BTM data and assimilated into the model. The measurements from BTM are first filtered with a bandpass filter to remove the periodic tidal signals, and then Ekman currents are obtained by removing the geostrophic component by using the assumption that the geostrophic current is assumed to be the sum of a constant reference velocity, and a component arising from a constant geostrophic shear. The modeled Ekman currents coincide well with the observed ones, especially for the upper layers. Compared with the assumption of constant and depth-dependent VEVCs, the results by using time-varying VEVC are the best, which also demonstrate the effectiveness and reasonability of this method. The sensitivity of estimation results to different kinds of wind drag coefficient formulae is also studied. The results indicate that the selection of wind drag coefficient formulae will not significantly influence the estimation of VEVCs under relative weak wind field. However, for large wind speed, such as during typhoon or hurricane, the selection of wind drag coefficient formulae might have a significant influence on the estimation of VEVCs.

The feasibility of typical Ekman model, the imperfection of balance equations, and the deficiencies of the present method are discussed in section 5. In this work, although the typical Ekman model is used, the VEVC is not a constant but estimated by assimilating in situ observations. If we take the ocean as a complex system which is driven by different forces, the in situ observations are actually the outputs of this system. The observations (output) are generated by various factors, including stratification, wind, horizontal pressure gradient, etc. As a result, the VEVCs estimated by assimilating in situ observations are in 
fact the implicit functions of these factors. It is an advantage of this methodology, which is based on the theory of inverse problem and parameter estimation, and can be taken as an improved method compared with the traditional parameterization. This work extends our understanding of Ekman layer model and provides a possible way to determine the temporal variations of VEVCs. Further work will concentrate on parameter estimation problems during typhoon or hurricane events, and discuss the temporal variations of VEVCs in the real ocean under the influence of strong wind forcing.

Acknowledgments. The authors thank the seven reviewers for the constructive suggestions which have greatly improved the manuscript. Financial support is provided by the National Key Research and Development Plan of China (Grants 2017YFA0604100 and 2017YFC1404000), the National Natural Science Foundation of China (Grants 41876086 and 41806012), Scientific Research Fund of the Second Institute of Oceanography, MNR (Grant JG1819), and the Fundamental Research Funds for the Central Universities of China. Jicai thanks the support of China Scholarship Council for the visiting research in WHOI, and he also thanks the host of WHOI. BTM data are provided by Ocean Physics Laboratory, University of California, Santa Barbara (http://opl.ucsb.edu).

\section{REFERENCES}

Alekseev, A. K., and I. M. Navon, 2001: The analysis of an ill-posed problem using multiscale resolution and second order adjoint techniques. Comput. Methods Appl. Mech. Eng., 190, 19371953, https://doi.org/10.1016/S0045-7825(00)00299-1.

,,-- and J. L. Steward, 2009: Comparison of advanced largescale minimization algorithms for the solution of inverse illposed problems. J. Optim. Meth. Software, 24, 63-87, https:// doi.org/10.1080/10556780802370746.

Anderson, D. L. T., J. Sheinbaum, and K. Hainesm, 1996: Data assimilation in ocean models. Rep. Prog. Phys., 59, 1209-1266, https://doi.org/10.1088/0034-4885/59/10/001.

Black, W. J., and T. D. Dickey, 2008: Observations and analyses of upper ocean responses to tropical storms and hurricanes in the vicinity of Bermuda. J. Geophys. Res., 113, C08009, https:// doi.org/10.1029/2007JC004358.

Cao, A. Z., H. Chen, W. Fan, H. L. He, J. B. Song, and J. C. Zhang, 2017: Estimation of eddy viscosity profile in the bottom Ekman boundary layer. J. Atmos. Oceanic Technol., 34, 21632175, https://doi.org/10.1175/JTECH-D-17-0064.1.

Chen, H. B., C. Miao, and X. Lv, 2013: Estimation of open boundary conditions for an internal tidal model with adjoint method: A comparative study on optimization methods. Math. Probl. Eng., 2013, 802136, https://doi.org/ 10.1155/2013/802136.

— A. Cao, J. Zhang, C. Miao, and X. Lv, 2014: Estimation of spatially varying open boundary conditions for a numerical internal tidal model with adjoint method. Math. Comput. Simul., 97, 14-38, https://doi.org/10.1016/j.matcom.2013.08.005.

Chereskin, T. K., 1995: Direct evidence for an Ekman balance in the California Current. J. Geophys. Res., 100, 18 261-18269, https://doi.org/10.1029/95JC02182.

Dattner, I., 2015: A model-based initial guess for estimating parameters in systems of ordinary differential equations. Biometrics, 71, 1176-1184, https://doi.org/ 10.1111/biom.12348.

Davies, A. M., and J. Xing, 2001: The influence of eddy viscosity parameterization and turbulence energy closure scheme upon the coupling of tidal and wind induced currents. Estuarine Coastal Shelf Sci., 53, 415-436, https://doi.org/10.1006/ ecss.1999.0623.

De Serio, F., and M. Mossa, 2015: Analysis of mean velocity and turbulence measurements with ADCPs. Adv. Water Resour., 81, 172-185, https://doi.org/10.1016/j.advwatres.2014.11.006.

Dickey, T., and Coauthors, 2001: Physical and biogeochemical variability from hours to years at the Bermuda Testbed Mooring site: June 1994-March 1998. Deep-Sea Res. II, 48, 2105-2140, https://doi.org/10.1016/S0967-0645(00)00173-9.

Edson, J. B., and Coauthors, 2013: On the exchange of momentum over the open ocean. J. Phys. Oceanogr., 43, 1589-1610, https://doi.org/10.1175/JPO-D-12-0173.1.

Ekman, V. W., 1905: On the influence of the Earth's rotation on ocean-currents. Ark. Mat. Astron. Fys., 2, 1-52.

Elipot, S., and S. T. Gille, 2009: Ekman layers in the Southern Ocean: Spectral models and observations, vertical viscosity and boundary layer depth. Ocean Sci., 5, 115-139, https:// doi.org/10.5194/os-5-115-2009.

Foreman, M. G. G., and R. F. Henry, 1989: The harmonic analysis of tidal model time series. Adv. Water Resour., 12, 109-120, https://doi.org/10.1016/0309-1708(89)90017-1.

Foreman, R. J., and S. Emeis, 2010: Revisiting the definition of the drag coefficient in the marine atmospheric boundary layer. J. Phys. Oceanogr., 40, 2325-2332, https://doi.org/ 10.1175/2010JPO4420.1.

Gwyther, D. E., B. K. Galton-Fenzi, M. S. Dinniman, J. L. Roberts, and J. R. Hunter, 2015: The effect of basal friction on melting and freezing in ice shelf-ocean models. Ocean Modell., 95, 3852, https://doi.org/10.1016/j.ocemod.2015.09.004.

Hager, W. W., and H. Zhang, 2005: A new conjugate gradient method with guaranteed descent and efficient line search. SIAM J. Optim., 16, 170-192, https://doi.org/ $10.1137 / 030601880$

Hui, Z., and Y. Xu, 2016: The impact of wave-induced CoriolisStokes forcing on satellite-derived ocean surface currents. J. Geophys. Res. Oceans, 121, 410-426, https://doi.org/10.1002/ 2015JC011082.

Hülsmann, M., T. Köddermann, J. Vrabec, and D. Reith, 2010: GROW: A gradient-based optimization workflow for the automated development of molecular models. Comput. Phys. Commun., 181, 499-513, https://doi.org/10.1016/ j.cpc.2009.10.024.

Jarosz, E., D. A. Mitchell, D. W. Wang, and W. J. Teague, 2007: Bottom-up determination of air-sea momentum exchange under a major tropical cyclone. Science, 315, 1707-1709, https://doi.org/10.1126/science.1136466.

Jiang, S., T. D. Dickey, D. K. Steinberg, and L. P. Madin, 2007: Temporal variability of zooplankton biomass from ADCP backscatter time series data at the Bermuda Testbed Mooring site. Deep-Sea Res. I, 54, 608-636, https://doi.org/10.1016/ j.dsr.2006.12.011. 
Jin, G., Q. Liu, and X. Lv, 2015: Inversion study of vertical eddy viscosity coefficient based on an internal tidal model with the adjoint method. Math. Probl. Eng., 2015, 915793, https:// doi.org/10.1155/2015/915793.

Jung, K., H. Kang, H. Lee, and M. Kim, 2007: Ekman motion in shallow open sea in the presence of time-harmonic variation of water depth. Cont. Shelf Res., 27, 1287-1302, https://doi.org/ 10.1016/j.csr.2007.01.022.

Kazantsev, E., 2012: Sensitivity of a shallow-water model to parameters. Nonlinear Anal. Real World Appl., 13, 1416-1428, https://doi.org/10.1016/j.nonrwa.2011.11.006.

Kirincich, A. R., and J. A. Barth, 2009: Time-varying acrossshelf Ekman transport and vertical eddy viscosity on the inner shelf. J. Phys. Oceanogr., 39, 602-620, https://doi.org/ 10.1175/2008JPO3969.1.

Kukulka, T., and K. Brunner, 2015: Passive buoyant tracers in the ocean surface boundary layer: 1 . Influence of equilibrium wind-waves on vertical distributions. J. Geophys. Res., 120, 3837-3858, https://doi.org/10.1002/2014JC010487.

Lacy, J. R., C. R. Sherwood, D. J. Wilson, T. A. Chisholm, and G. R. Gelfenbaum, 2005: Estimating hydrodynamic roughness in a wave-dominated environment with a high-resolution acoustic Doppler profiler. J. Geophys. Res., 110, C06014, https://doi.org/10.1029/2003JC001814.

Lardner, R. W., and Y. Song, 1995: Optimal estimation of Eddy viscosity and friction coefficients for a quasi-threedimensional numerical tidal model. Atmos.-Ocean, 33 581-611, https://doi.org/10.1080/07055900.1995.9649546.

Large, W. G., and S. Pond, 1981: Open ocean momentum flux measurements in moderate to strong winds. J. Phys. Oceanogr., 11, 324-336, https://doi.org/10.1175/1520-0485(1981)011<0324: OOMFMI $>2.0 . \mathrm{CO} ; 2$.

_- and S. G. Yeager, 2009: The global climatology of an interannually varying air-sea flux data set. Climate Dyn., 33, 341364, https://doi.org/10.1007/s00382-008-0441-3.

_ - J. C. Mcwilliams, and S. C. Doney, 1994: Oceanic vertical mixing: A review and a model with a nonlocal boundary layer parameterization. Rev. Geophys., 32, 363-403, https://doi.org/ 10.1029/94RG01872.

Launder, B. E., G. J. Reece, and W. Rodi, 1975: Progress in the development of a Reynolds-stress turbulence closure. J. Fluid Mech., 68, 537-566, https://doi.org/10.1017/ S0022112075001814.

Lenn, Y. D., and T. K. Chereskin, 2009: Observations of Ekman currents in the Southern Ocean. J. Phys. Oceanogr., 39, 768779, https://doi.org/10.1175/2008JPO3943.1.

Lentz, S. J., 1995: Sensitivity of the inner-shelf circulation to the form of the eddy viscosity profile. J. Phys. Oceanogr., 25, 19-28, https://doi.org/10.1175/1520-0485(1995)025<0019: SOTISC $>2.0 . \mathrm{CO} ; 2$.

Lewis, D. M., and S. E. Belcher, 2004: Time-dependent, coupled, Ekman boundary layer solutions incorporating Stokes drift. Dyn. Atmos. Oceans, 37, 313-351, https://doi.org/10.1016/ j.dynatmoce.2003.11.001.

Liu, D. C., and J. Nocedal, 1989: On the limited memory BFGS method for large scale optimization. Math. Program., 45, 503528, https://doi.org/10.1007/BF01589116.

Liu, B., K. Wu, and C. Guan, 2007: Global estimates of wind energy input to subinertial motions in the Ekman-Stokes layer. J. Oceanogr., 63, 457-466, https://doi.org/10.1007/s10872-0070041-6.

Liu, J., J. Dai, D. Xu, J. Wang, and Y. Yuan, 2018: Seasonal and interannual variability in coastal circulations in the northern
South China Sea. Water, 10, 520, https://doi.org/10.3390/ w10040520.

Lozovatsky, I., Z. Liu, H. Wei, and H. J. S. Fernando, 2008: Tides and mixing in the northwestern East China Sea. Part II: Nearbottom turbulence. Cont. Shelf Res., 28, 338-350, https:// doi.org/10.1016/j.csr.2007.08.007.

Lu, X., and J. Zhang, 2006: Numerical study on spatially varying bottom friction coefficient of a 2D tidal model with adjoint method. Cont. Shelf Res., 26, 1905-1923, https://doi.org/ 10.1016/j.csr.2006.06.007.

Maurer, K. D., G. Bohrer, W. Kenny, and V. Ivanov, 2015: Largeeddy simulations of surface roughness parameter sensitivity to canopy-structure characteristics. Biogeosciences, 12, 25332548, https://doi.org/10.5194/bg-12-2533-2015.

McWilliams, J. C., E. Huckle, and A. F. Shchepetkin, 2009: Buoyancy effects in a stratified Ekman layer. J. Phys. Oceanogr., 39, 2581-2599, https://doi.org/10.1175/2009JPO4130.1.

Mellor, G. L., and T. Yamada, 1982: Development of a turbulence closure model for geophysical fluid problems. Rev. Geophys. Space Phys., 20, 851-875, https://doi.org/10.1029/ RG020i004p00851.

Navon, I. M., 1998: Practical and theoretical aspects of adjoint parameter estimation and identifiability in meteorology and oceanography. Dyn. Atmos. Oceans, 27, 55-79, https://doi.org/ 10.1016/S0377-0265(97)00032-8.

, and D. M. Legler, 1987: Conjugate-gradient methods for large-scale minimization in meteorology. Mon. Wea. Rev., 115, 1479-1502, https://doi.org/10.1175/1520-0493(1987)115<1479: CGMFLS $>2.0 . \mathrm{CO} ; 2$

$\longrightarrow$, X. Zou, J. Derher, and J. Sela, 1992: Variational data assimilation with an adiabatic version of the NMC spectral model. Mon. Wea. Rev., 120, 1433-1446, https://doi.org/ 10.1175/1520-0493(1992)120<1433:VDAWAA > 2.0.CO;2.

Nystrom, E. A., C. R. Rehmann, and M. Asce, 2007: Evaluation of mean velocity and turbulence measurements with ADCPs. J. Hydraul. Eng., 133, 1310-1318, https://doi.org/10.1061/ (ASCE)0733-9429(2007)133:12(1310).

Obermeier, F., 2006: Prandtl's mixing length model - Revisited. Proc. Appl. Math. Mech., 6, 577-578, https://doi.org/10.1002/ pamm.200610269.

Pacanowski, R. C., and S. G. H. Philander, 1981: Parameterization of vertical mixing in numerical models of tropical oceans. J. Phys. Oceanogr., 11, 1443-1451, https://doi.org/10.1175/ 1520-0485(1981)011<1443:POVMIN>2.0.CO;2.

Panchang, V. G., and J. E. Richardson, 1993: Inverse adjoint estimation of eddy viscosity for coastal flow models. J. Hydraul. Eng., 119, 506-524, https://doi.org/10.1061/ (ASCE)0733-9429(1993)119:4(506).

Peng, S., and Y. Li, 2015: A parabolic model of drag coefficient for storm surge simulation in the South China Sea. Sci. Rep., 5, 15 496, https://doi.org/10.1038/srep15496.

Phillips, H. E., and N. L. Bindoff, 2014: On the nonequivalent barotropic structure of the Antarctic Circumpolar Current: An observational perspective. J. Geophys. Res. Oceans., 119, 5221-5243, https://doi.org/10.1002/2013JC009516.

Pohlmann, T., 1996: Calculating the annual cycle of the vertical eddy viscosity in the North Sea with a three-dimensional baroclinic shelf sea circulation model. Cont. Shelf Res., 16, 147-161, https://doi.org/10.1016/0278-4343(94)E0037-M.

Polton, J. A., D. M. Lewis, and S. E. Belcher, 2005: The role of wave-induced Coriolis-Stokes forcing on the wind-driven mixed layer. J. Phys. Oceanogr., 35, 444-457, https://doi.org/ 10.1175/JPO2701.1. 
— - Y. Lenn, S. Elipot, T. K. Chereskin, and J. Sprintall, 2013: Can Drake Passage observations match Ekman's classic theory. J. Phys. Oceanogr., 43, 1733-1740, https://doi.org/10.1175/ JPO-D-13-034.1.

Price, J. F., and M. A. Sundermeyer, 1999: Stratified Ekman layers. J. Geophys. Res., 104, 20 467-20 494, https://doi.org/10.1029/ 1999JC900164.

_, R. A. Weller, and R. R. Schudlich, 1987: Wind-driven ocean currents and Ekman transport. Science, 238, 1534-1538, https://doi.org/10.1126/science.238.4833.1534.

Ralph, E. A., and P. P. Niiler, 1999: Wind-driven currents in the tropical Pacific. J. Phys. Oceanogr., 29, 2121-2129, https://doi.org/ 10.1175/1520-0485(1999)029<2121:WDCITT > 2.0.CO;2.

Roach, C. J., H. E. Phillips, N. L. Bindoff, and S. R. Rintoulet, 2015: Detecting and characterizing Ekman currents in the Southern Ocean. J. Phys. Oceanogr., 45, 1205-1223, https://doi.org/ 10.1175/JPO-D-14-0115.1.

Shanno, D. F., and K. H. Phua, 1980: Remark on "Algorithm 500: Minimization of Unconstrained Multivariate Functions [E4]". ACM Trans. Math. Software, 6, 618-622, https://doi.org/ 10.1145/355921.355933.

Song, J. B., 2009: The effects of random surface waves on the steady Ekman current solutions. Deep-Sea Res. I, 56, 659-671, https:// doi.org/10.1016/j.dsr.2008.12.014.

Thacker, W. C., and R. B. Long, 1988: Fitting dynamics to data. J. Geophys. Res., 93, 1227-1240, https://doi.org/10.1029/ JC093iC02p01227.

Tikhonov, A. N., 1963: Solution of incorrectly formulated problems and the regularization method. Soviet Math.s Dokl., 4, $1035-1038$.

Wang, Z., I. M. Navon, F. X. Le Dimet, and X. Zou, 1992: The second order adjoint analysis: Theory and applications. Meteor. Atmos. Phys., 50, 3-20, https://doi.org/10.1007/BF01025501.

,,-- X. Zou, and F. X. Le Dimet, 1995: A truncated Newton optimization algorithm in meteorology applications with analytic Hessian vector products. Comput. Optim. Appl., 4, 241262, https://doi.org/10.1007/BF01300873.

Wirth, A., 2010: On the Ekman spiral with an anisotropic eddy viscosity. Bound.-Layer Meteor., 137, 327-331, https://doi.org/ 10.1007/s10546-010-9527-7.

$\mathrm{Wu}, \mathrm{K}$., and B. Liu, 2008: Stokes drift-induced and direct wind energy inputs into the Ekman layer within the Antarctic Circumpolar Current. J. Geophys. Res., 113, C10002, https:// doi.org/10.1029/2007JC004579.

Xu, Y., H. He, J. Song, Y. Hou, and F. Li, 2017: Observations and modeling of typhoon waves in the South China Sea. J. Phys. Oceanogr., 47, 1307-1324, https://doi.org/10.1175/ JPO-D-16-0174.1.

Yoshikawa, Y., and T. Endoh, 2015: Estimating the eddy viscosity profile from velocity spirals in the Ekman boundary layer.
J. Atmos. Oceanic Technol., 32, 793-804, https://doi.org/ 10.1175/JTECH-D-14-00090.1.

, T. Matsuno, T. Wagawa, E. Tsutsumi, H. Yoshimura, and Y. Morii, 2010: Turbulent bottom Ekman boundary layer measured over a continental shelf. Geophys. Res. Lett., 37, L15605, https://doi.org/10.1029/2010GL044156.

Yu, L., and J. J. O'Brien, 1991: Variational estimation of the wind stress drag coefficient and the oceanic eddy viscosity profile. J. Phys. Oceanogr., 21, 709-719, https://doi.org/ 10.1175/1520-0485(1991)021<0709:VEOTWS > 2.0.CO;2.

Zedler, S. E., T. D. Dickey, S. C. Doney, J. F. Price, X. Yu, and G. L. Mellor, 2002: Analyses and simulations of the upper ocean's response to Hurricane Felix at the Bermuda Testbed Mooring site: 13-23 August 1995. J. Geophys. Res., 107, 3232, https://doi.org/10.1029/2001JC000969.

Zhang, J., and X. Lu, 2008: Parameter estimation for a threedimensional numerical barotropic tidal model with adjoint method. Int. J. Numer. Methods Fluids, 57, 47-92, https:// doi.org/10.1002/fld.1620.

- , and - 2010: Inversion of three-dimensional tidal currents in marginal seas by assimilating satellite altimetry. Comput. Methods Appl. Mech. Eng., 199, 3125-3136, https://doi.org/ 10.1016/j.cma.2010.06.014.

_ , and Y. Wang, 2014: A method for inversion of periodic open boundary conditions in two-dimensional tidal models. Comput. Methods Appl. Mech. Eng., 275, 20-38, https://doi.org/ 10.1016/j.cma.2014.02.020.

— X. Xu, P. Wang, and Y. P. Wang, 2011: Study on linear and nonlinear bottom friction parameterizations for regional tidal models using data assimilation. Cont. Shelf Res., 31, 555-573, https://doi.org/10.1016/j.csr.2010.12.011.

Zhang, Q., Y. Gao, and X. Lv, 2015: Estimation of oceanic eddy viscosity profile and wind stress drag coefficient using adjoint method. Math. Probl. Eng., 2015, 309525, https://doi.org/ 10.1155/2015/309525.

Zhang, Y., J. Tian, and L. Xie, 2009: Estimation of eddy viscosity on the South China Sea shelf with adjoint assimilation method. Acta Oceanol. Sin., 28, 9-16.

Zhu, Y., and I. M. Navon, 1999: Impact of parameter estimation on the performance of the FSU global spectral model using its fullphysics adjoint. Mon. Wea. Rev., 127, 1497-1517, https://doi.org/ 10.1175/1520-0493(1999)127<1497:IOPEOT>2.0.CO;2.

Zijlema, M., G. P. Van Vledder, and L. H. Holthuijsen, 2012: Bottom friction and wind drag for wave models. Coast. Eng., 65, 19-26, https://doi.org/10.1016/j.coastaleng.2012.03.002.

Zou, X., I. M. Navon, M. Berger, P. K. H. Phua, T. Schlick, and F. X. Le Dimet, 1993: Numerical experience with limited-memory quasi-Newton methods and truncated Newton methods. SIAM J. Numer. Optim., 3, 582-608, https://doi.org/10.1137/0803029. 